\title{
Applying Explicit Schemes to the Korteweg-de Vries Equation
}

\author{
Masitah Shahrill ${ }^{1}$, Maureen Siew Fang Chong ${ }^{1} \&$ Hajah Norhakimah Haji Mohd Nor ${ }^{2}$ \\ ${ }^{1}$ Sultan Hassanal Bolkiah Institute of Education, Universiti Brunei Darussalam, Bandar Seri Begawan, Brunei \\ Darussalam \\ ${ }^{2}$ Sultan Bolkiah Vocational School, Ministry of Education, Bandar Seri Begawan, Brunei Darussalam \\ Correspondence: Masitah Shahrill, Sultan Hassanal Bolkiah Institute of Education, Universiti Brunei Darussalam, \\ Jalan Tungku Link, Gadong, BE 1410, Bandar Seri Begawan, Brunei Darussalam. Tel: 673-246-3001. Fax: \\ 673-246-1003. E-mail: masitah.shahrill@ubd.edu.bn
}

Received: October 26, 2014

Accepted: November 15, 2014 Online Published: March 4, 2015

doi:10.5539/mas.v9n4p200

URL: http://dx.doi.org/10.5539/mas.v9n4p200

\begin{abstract}
The water wave soliton is a result of a dynamic balance between dispersion and nonlinear effects. It brings together many branches of mathematics, some of which touch on deep ideas. The Korteweg-de Vries equation is typical of all model equations of nonlinear waves in the soliton phenomena. Four explicit difference schemes are used in order to approximate the Korteweg-de Vries equation, namely; (a) a First order upwind scheme, (b) the Zabusky-Kruskal scheme, (c) the Lax-Wendroff scheme, and (d) the Walkley scheme. Our main interest was to analyse which explicit scheme among the four performs well when implemented to the KdV equation to produce the best soliton solution. Hence, reviewing and considering existing schemes. Three sets of initial data are used to explore the numerical approximations. Two, including the data proposed by Zabusky and Kruskal, involve a single soliton wave, whilst the other involves the separation into two solitons, which will interact in time. These initial conditions and periodic boundary conditions are described in detail taking into account physical, mathematical and computational considerations. Accuracy, consistency and Fourier stability in regard to the four explicit schemes for the Korteweg-de Vries equation are discussed. Numerical results are reported for a single soliton solution and the separation into two solitons with different velocities are investigated. Graphical results are presented to show how well these four schemes agree well with each other. After comparing the four explicit schemes, the best scheme was the Zabusky and Kruskal scheme since it is a two-step scheme, which uses the explicit leapfrog finite difference scheme and was good for low amplitudes and less running time was needed than the other three explicit schemes.
\end{abstract}

Keywords: Korteweg-de Vries equation, explicit difference schemes, solitons

\section{Introduction}

Travelling waves solutions to the Korteweg-de Vries equation, which is a nonlinear Partial Differentiation equation of third order, have been of some interest for 150 years. In the Korteweg-de Vries equation, two different mechanisms, i.e., nonlinearity and dispersion are present, and a remarkable property of this equation is that the balance between these two mechanisms will produce solitary waves. When several solitary waves are present they are termed Solitons.

Soliton theory developed after the discovery by Gardner, Greene, Kruskal and Miura (1967) of the Inverse Scattering Transform for the Korteweg-de Vries equation (see equation (2.2)). They were led to this by the earlier discovery of solitons by Zabusky and Kruskal (1965), who were studying the Fermi-Pasta-Ulam problem of 1-dimensional lattices. This is a model, which turns out to be closely related to a discretisation of the Korteweg-de Vries equation, where there was no equipartition of energy among the modes. Thus started the modern development of soliton theory.

The theory of solitons is attractive and exciting. It has an interesting history as described in Sections 2.1 and 2.2 and looks to set a promising future for twenty-first century mathematics. In addition, in Section 2.3 we provide an introduction to the Korteweg-de Vries equation and how we can imagine that it is the simplest equation, which incorporated both nonlinearity and dispersion. In this section, applications of the Korteweg-de Vries equation are described and an analytical exact result to the Korteweg-de Vries equation by means of elementary operations is presented. 
The numerical modelling of the one-dimensional form of the Korteweg-de Vries equation is considered in Section 3. Here, the four explicit finite difference methods are listed. Three sets of initial data are investigated. Two involve single solitons (including the data proposed by Zabusky and Kruskal (1965)) whilst the other involves two solitons separating, which will later collide. Different values of parameters present in the third initial data are explored. Attention is paid to the initial conditions and periodic boundary conditions for all the schemes, and the techniques that are described will enable us to relate and apply them in Section 4. Subsequently, in Section 4, we introduce in more detail the four explicit schemes used in this study. They are a First Order Upwind Scheme, the Zabusky and Kruskal scheme, the Lax-Wendroff scheme and the Walkley scheme. Their properties are investigated and their applications to the Korteweg-de Vries equation will be shown. For each section, graphical results are shown together with the comments.

The Korteweg-de Vries equation has been extensively studied in numerous studies using symplectic and multisymplectic methods (Ascher \& McLachlan, 2005; Wang, Wang \& Hu, 2008; Lv, Xue \& Wang, 2011; Dutykh, Chhay \& Fedele, 2013), and the space and time-accurate numerical method (Kumar \& Mehra, 2005), entirely to demonstrate the accuracy and stability of the dynamic of the equation. Consequently, multisymplectic schemes are most accurate and stay remarkably stable over a long period of time but is somewhat slow (Wang et al., 2008). Indeed, there are many new explicit schemes for the Korteweg-de Vries equation (see also Akdi \& Sedra, 2013a, 2013b), our main interest was to analyse which explicit existing scheme among the four: (a) a First order upwind scheme, (b) the Zabusky-Kruskal scheme, (c) the Lax-Wendroff scheme, and (d) the Walkley scheme performs well when implemented to the KdV equation to produce the best soliton solution.

Consequently, the sources used for this study are mainly from the book by Drazin and Johnson (1989), a journal by Taha and Ablowitz (1984) where we followed their initial conditions for the 1 - Soliton solution and the initial condition for the $2-$ Soliton solution. The initial condition used for the cos function and the Zabusky and Kruskal (1965). For the Walkley scheme, we followed the work of M. Walkley (1999), where a scheme to the Korteweg-de Vries equation was applied in connection to model the extended shallow-water wave equations. To summarise, the objective of this paper is to review and consider the four classical existing schemes for the Korteweg-de Vries equation for each of the schemes stated above.

\section{The History of Solitons and the Korteweg-de Vries Equation (KdV)}

\subsection{The Discovery of Solitary Waves}

The occurrence of a soliton was first recorded by a young Scottish civil engineer named John Scott Russell (1808-1882) on the side of the Union canal near Edinburg in 1834. Russell (1845) then reported his observations to the British Association in his 1844 'Report on waves' in the following now famous description:

I was observing the motion of a boat which was rapidly drawn along a narrow channel by a pair of horses, when the boat suddenly stopped - not so the mass of water in the channel which it had put in motion; it accumulated round the prow of the vessel in a state of violent agitation, then suddenly leaving it behind, rolled forward with great velocity, assuming the form of a large solitary elevation, a rounded, smooth and well-defined heap of water, which continued its course along the channel apparently without change of form or diminution of speed. I followed it on horseback, and overtook it still rolling on a rate of eight or nine miles an hour, preserving its original figure some thirty feet long and a foot to a foot and a half in height. Its height gradually diminished, and after a chase of one or two miles I lost it in the windings of the channel. Such, in the month of August 1834, was my first chance interview with that singular and beautiful phenomenon which I have called the Wave of Translation.

Following this scientific discovery, Scott Russell built a 30' wave tank in his back garden where he generated solitary waves by dropping a weight at one end of the water channel (see Figure 1.1).

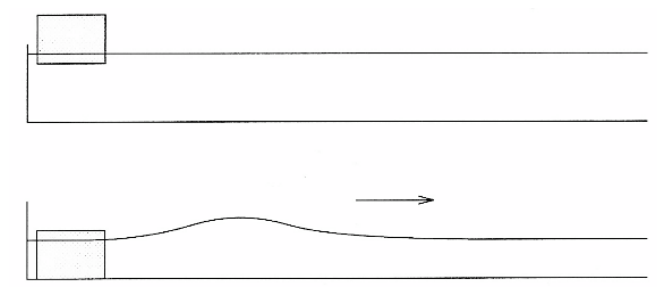

Figure 1.1. Scott Russell's experiment to generate a solitary wave 
He was able to deduce empirically that the volume of water displaced and found that the solitary wave is obtained from

$$
c^{2}=g(h+a)
$$

where $c$ is the speed, $a$ the amplitude of wave, $h$ the undisturbed depth of water and $g$ is the acceleration of gravity.

Therefore, he observed from Figure 1.2 that the solitary wave is a gravity wave.

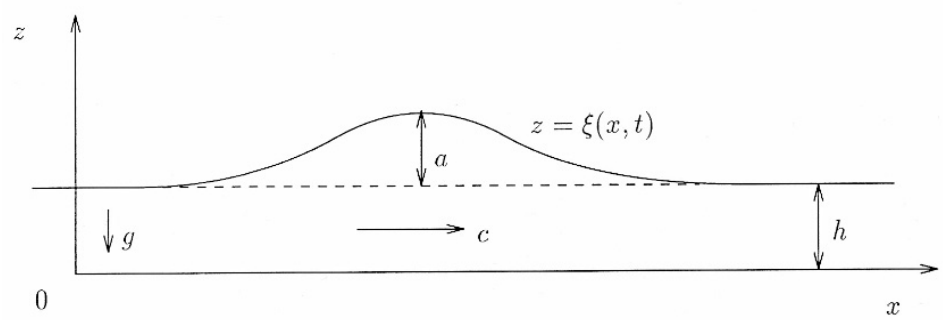

Figure 1.2. The parameters and variables used in the description of the solitary wave

John Valentin Boussinesq (1871) and John William Strutt or Lord Rayleigh (1876) both assumed that a solitary wave has a length scale much greater than the depth of the water. From the equations of motions of an inviscid incompressible fluid, they deduced Scott Russell's formula for $c$. They even concluded that the wave profile $z=\zeta(x, t)$ is given by

$$
\zeta(x, t)=a \operatorname{sech}^{2} \beta(x-c t)
$$

where $\beta^{-2}=\frac{4 h^{2}(h+a)}{3 a}$ for any $a>0$ and $\operatorname{sech}^{2}$ profile is strictly true only if $\frac{a}{h} \ll 1$.

The final step to get a solution out of equation (1.2) was finished off by Korteweg and de Vries in 1895 . Provided $\varepsilon$ and $\sigma$ are small

$$
\frac{\partial \zeta}{\partial t}=\frac{3}{2}\left(\frac{g}{h}\right)^{\frac{1}{2}}\left(\frac{2}{3} \varepsilon \frac{\partial \zeta}{\partial \chi}+\frac{1}{3} \sigma \frac{\partial^{3} \zeta}{\partial \chi^{3}}\right)
$$

where $\chi$ is a magnitude chosen to be moving with the waves.

Using the change of variables

$$
\zeta=\zeta(X, t), \quad X=\chi+\varepsilon\left(\frac{g}{h}\right)^{\frac{1}{2}} t
$$

equation (1.3) can be re-assigned as the Korteweg-de Vries (KdV) equation

$$
\zeta_{t}=\frac{3}{2}\left(\frac{g}{h}\right)^{\frac{1}{2}}\left(\zeta \zeta_{X}+\frac{1}{3} \sigma \zeta_{X X X}\right)
$$

The parameter $\sigma$ includes as a part of the surface tension, $T$, in the form $\sigma=\frac{1}{3} h^{3}-\frac{T h}{g \rho}$, where $\rho$ is the density and $\varepsilon$ is an arbitrary parameter. If the solution of equation (1.3) is stationary in the frame $\chi$ the $\zeta=\zeta(\chi)$, hence

$$
\frac{2}{3} \varepsilon \zeta^{\prime}+\zeta \zeta^{\prime}+\frac{1}{3} \sigma \zeta^{\prime \prime \prime}=0
$$

If $\zeta \rightarrow 0$ as $|\chi| \rightarrow \infty$, integrate (1.5) twice to produce

$$
2 \varepsilon \zeta^{2}+\zeta^{3}+\sigma(\zeta)^{2}=0
$$

It is easily verified by direct substitution that

$$
\zeta(\chi)=a \operatorname{sech}^{2}(\beta \chi)=0
$$

is a solution, provided 


$$
a=4 \sigma \beta^{2} \text { and } \varepsilon=-2 \sigma \beta^{2}
$$

The coordinate $\chi$ is defined (by Korteweg and de Vries, 1895) as

$$
\chi=x-(g h)^{\frac{1}{2}}\left(1-\frac{\epsilon}{h}\right) t
$$

Hence the solitary wave solution becomes

$$
\zeta(x, t)=a \operatorname{sech} \mid \frac{1}{2}\left(\frac{\alpha}{\sigma}\right)^{\frac{1}{2}}\left(x-(g h)^{\frac{1}{2}}\left(1+\frac{1}{2} \frac{a}{h}\right) t \mid\right.
$$

This agrees with equations (1.1) and (1.2), neglecting the surface tension, so $\sigma=\frac{1}{3} h^{3}$ and assuming $\frac{a}{h} \ll 1$, then

$$
c \sim(g h)^{\frac{1}{2}}\left(1+\frac{1}{2} \frac{a}{h}\right) \quad \text { and } \quad \beta \sim \frac{1}{2}\left(\frac{3 a}{h^{3}}\right)^{\frac{1}{2}}
$$

Therefore Scott Russel's solitary wave, equation (1.2) is a solution of the KdV equation. In conclusion, taller waves are narrower and travel faster.

\subsection{Interacting Solitary Waves}

It was not until the mid 1960's when applied scientists began to use modern digital computers to study wave propagation that the early ideas Scott Russell had begun to be appreciated. He viewed the solitary wave as a self-sufficient dynamic entity; a 'thing' displaying many properties of a particle. Hidden away in Scott Russell's 'Report on Waves' (1844, pg. 384, plate XLVII) (Russell, 1845) is the diagram reproduced in Figure 1.3.
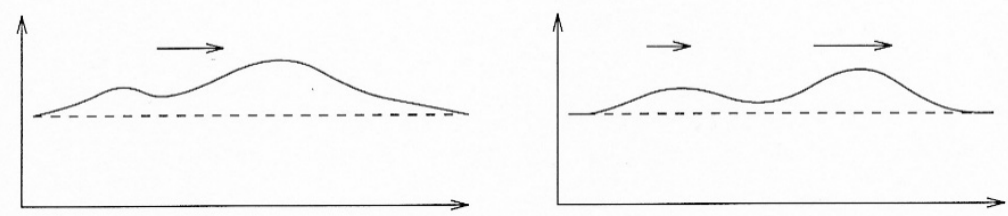

Figure 1.3. A Sketch of Scott Russell's ‘compound' wave

Scott Russell described this figure as "represents the genesis by a large low column of fluid of a compound or double wave of the first order, which immediately breaks down by spontaneous analysis into two, the greater moving faster and altogether leaving the smaller". (Note, the very slowly diminishing height of the water wave that he observed is due to 'frictional' losses and is not taken into account in the standard $\mathrm{KdV}$ equation).

If we start from the initial profile of Figure 1.3 but with the taller wave left of the shorter wave, then a remarkable property is observed as represented in Figure 1.4.
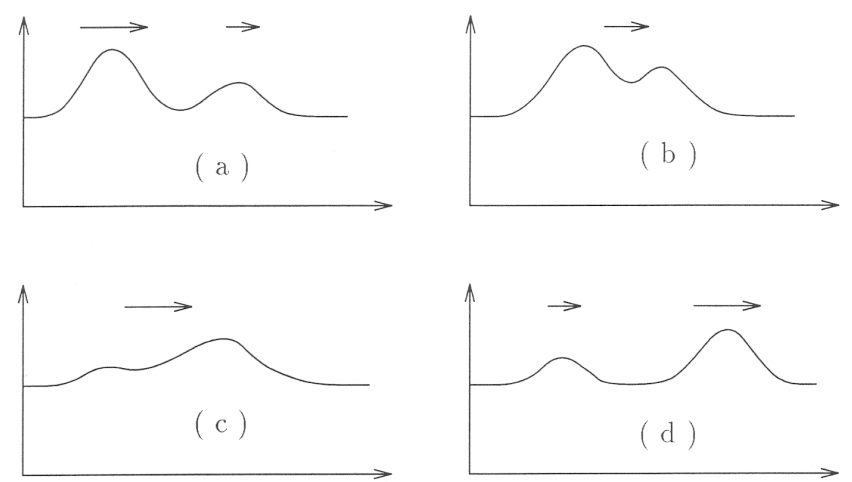

Figure 1.4. A Sketch depicting the interaction of two 'solitons'. For times (a) $t=t_{1}$, (b) $t=t_{2}>t_{1}$,

(c) $t=t_{3}>t_{2}$ and (d) $t=t_{1}>t_{3}$. 
From the sketch, the taller wave catches up, interacts with and then passes the shorter wave. It appears to be that the taller one is overtaking the shorter one and continues its path undamaged and undisturbed. Of course, this is to be expected if the two waves satisfies the Linear Superposition Principle, but in fact they do not.

In the late 1960's, Zabusky and Kruskal (1965) considered the initial value problem: $u_{t}+u u_{x}+\delta^{2} u_{x x x}=0$, (equation (3.1)) with periodic boundary conditions. They solved this KdV equation with the initial condition presented in equation (3.2). This Incredulous duo numerically discovered that the KdV solitary waves maintained their identity following collisions and reporting that "here we have a nonlinear physical process in which interacting localized pulses do not scatter irreversibly".

This persistence of the wave led by Zabusky and Kruskal to coin the term 'Soliton' to emphasize the particle-like nature of these robust travelling solitary waves. The Zabusky and Kruskal Scheme will be discussed and their results shown in Section 4.2.

Solitons are very stable solitary waves in the solution of many model equations of nonlinear phenomena. When solitons are located mutually far apart, each of them is approximately a travelling wave with constant shape and velocity. As two such solitary waves get closer, they gradually deform and finally merge into a single wave packet, which, however soon splits into two solitary waves with the same shape and velocity before the 'collision'. In fact, a soliton is very robust against perturbations, the bottom of the canal may be uneven and bumpy, ducks and dogs may swim around in the canal but the soliton will gently pass these obstacles intact and undistorted.

\subsection{The Korteweg-de Vries Equation (KdV)}

In 1895, two Dutchmen, Korteweg and de Vries (1895) derived a nonlinear partial differential equation (the so called $\mathrm{KdV}$ equation) of the form

$$
u_{t}+u u_{x}+\delta^{2} u_{x x x}=0, \quad(\mu>0)
$$

which describes the long time asymptotic behaviour of small but finite amplitude of one-dimensional shallow water waves. In the equation above $u \equiv u(x, t)$ measures the elevation (the height of water above the equilibrium level) at time $t$ and position $x$. The subscripts denote partial differentiation. In the KdV equation (2.1), two different mechanisms are present, i.e.

1. Non-linearity $\left(u u_{x}\right)$, which tends to steepen those parts having negative slope,

2. Dispersion $\left(u_{x x x}\right)$, which makes dispersive wave components of different wave numbers (frequencies) propagate at different velocities.

The delicate balance between these two effects leads to travelling wave of permanent form, the so-called Solitary Wave. It is usual to refer to the Solitary Wave as the single-soliton solution, but when more than one of them appears in a solution they are then termed as Solitons or 'Solitary-wave pulses'. If one of these two competing effects is lost, solitons become unstable and, eventually, cease to exist. In this respect, solitons are completely different from linear waves.

The KdV equation has also been found to describe a number of important physical phenomena such as magnetohydrodynamic waves in a warm plasma (Kakutani, Kawahara \& Taniuti, 1967), acoustic waves in an anharmonic crystal (Zabusky, 1967), ion-acoustic waves in a plasma (Washimi \& Taniuti, 1966), the long lived 'giant red spot' in the highly turbulent Jovian atmosphere and the propagation of short laser pulses in optical fibers (Hasegawa, 1990).

\subsubsection{Applications of the KdV Equation}

Consider a linear wave motion in one dimension with dispersion. The KdV equation combines the linear dispersion relation of the form

$$
w(k)=k c\left(k^{2}\right)
$$

with a typical nonlinear convection operator. It must take this form because only odd derivatives of $u$ are present.

For infinitely long waves $(k \rightarrow 0)$

$$
\frac{w}{k} \sim c_{0}-\lambda k^{2}
$$

where $c_{0}$ is a non-zero speed of propagation, and since long waves travel fastest, then $\lambda>0$. This dispersion relation is obtained from the equation

$$
u_{t}+c_{0} u_{x}+\lambda u_{x x x}=0
$$


If the medium where the transmission is occurring is a classical continuum, then the time evolution will be given by the material derivative

$$
\frac{D}{D t}=\frac{\partial}{\partial t}+u\left(\frac{\partial}{\partial x}\right)
$$

If these two effects balance, then

$$
u_{\tau}+u u_{\xi}+\lambda u_{\xi \xi \xi}=0, \quad \xi=x-c_{0} t, \quad \tau=\alpha t
$$

This is valid in a suitable region of the $(x, t)$ plane, described precisely by $x-c_{0} t=O(1), t=O\left(\alpha^{-1}\right)$ as $\alpha \rightarrow 0$.

Equation (2.1), which describes the (unidirectional) propagation of waves on the surface of a shallow channel studied by Korteweg and de Vries (1895) late in the $19^{\text {th }}$ Century (after performing a Galilean and a variety of scaling transformations), can be formulated in simplified form

$$
u_{t}(x, t)+6 u(x, t) u_{x}(x, t)+u_{x x x}(x, t)=0
$$

or

$$
u_{t}+6 u u_{x}+u_{x x x}=0
$$

This KdV equation is the champion of model equations of nonlinear waves. It is nonlinear because of the product shown in the second summand and of third order for the reason that the third derivative is highest. The factor 6 is just a scaling factor to make the solutions (solitons or solitary waves) easier to describe.

\subsubsection{Exact Solution to the KdV Equation}

The aim here is to find general exact solutions to the $\mathrm{KdV}$ equation (2.2) where neither initial conditions nor boundary conditions are to be used yet. Seek a travelling wave solution of the form

$$
u(x, t)=f(z), \quad z=\xi-\beta \tau
$$

i.e.

$$
u(x, t)=f(\xi-\beta \tau)
$$

with $f, f^{\prime}, f^{\prime \prime} \rightarrow 0$ as $|z| \rightarrow \infty$.

Substituting the solution (2.3) into (2.2) leads to the Ordinary Differential Equation

$$
-\beta f^{\prime}(z)+6 f(z) f^{\prime}(z)+f^{\prime \prime \prime}(z)=0
$$

Integration can be done directly since equation (2.4) is in the form of a total derivative. It follows from (2.4) that

$$
-\beta f+3 f^{2}+f^{\prime \prime}=0
$$

where the integration constant vanishes due to decay conditions of $f$.

Multiplying by $f^{\prime}$

$$
-\beta f f^{\prime}+3 f^{2} f^{\prime}+f^{\prime} f^{\prime \prime}=0
$$

allows us to integrate to obtain

$$
-\frac{1}{2} \beta f^{2}+f^{3}+\frac{1}{2}\left(f^{\prime}\right)^{2}=0
$$

Again, here the constant disappears.

With constants equal to zero, equation (2.5) can be written as

$$
\left(f^{\prime}\right)^{2}=\beta f^{2}-2 f^{3}=f^{2}(\beta-2 f)
$$

Notice that a real solution exists only if $\left(f^{\prime}\right)^{2} \geq 0$, i.e. if $\beta-2 f \geq 0$.

$$
\frac{d f}{d z}=\sqrt{f^{2}(\beta-2 f)}= \pm f \sqrt{\beta-2 f}
$$

By separation of variables

$$
f \frac{d f}{f \sqrt{\beta-2 f}}= \pm\left(z-z_{0}\right) .
$$


To evaluate the integral on the left hand side of (2.6), we use the substitution

$$
f=\frac{1}{2} \beta \operatorname{sech}^{2} w
$$

Then

$$
\begin{aligned}
\frac{d f}{d z} & =(\beta \operatorname{sech} w)(-\operatorname{sech} w \tanh w) \\
& =-\beta \operatorname{sech}^{2} w \tanh w
\end{aligned}
$$

and

$$
\begin{aligned}
I & =\int \frac{d f}{d \sqrt{\beta-2 f}}, \\
& =-\int \frac{\beta \operatorname{sech}^{2} w \tanh w d w}{\frac{1}{2} \beta \operatorname{sech}^{2} w \sqrt{\beta\left(1-\operatorname{sech}^{2} w\right)}}, \\
& =-\int \frac{\tanh w d w}{\frac{1}{2} \sqrt{\beta} \tanh w}, \\
& =-\frac{2}{\sqrt{\beta}} w .
\end{aligned}
$$

Hence:

$$
\begin{aligned}
-\frac{2}{\sqrt{\beta}} w & = \pm\left(z-z_{0}\right), \\
w & =\mp \frac{\sqrt{\beta}}{2}\left(z-z_{0}\right), \\
f & =\frac{1}{2} \beta \operatorname{sech}^{2} w \\
f(z) & =\frac{1}{2} \beta \operatorname{sech}^{2} w \frac{\sqrt{\beta}}{2}\left(z-z_{0}\right) .
\end{aligned}
$$

Transform back to $u(\xi, \tau)=f(z)$ where $z=\xi-c \tau$ and obtain

$$
f(z)=\frac{1}{2} \beta \operatorname{sech}^{2} w \frac{\sqrt{\beta}}{2}\left(\xi-\beta \tau-z_{0}\right) .
$$

Comparing equation (2.7) and Taha and Ablowitz (1984), we finally get

where

$$
u(x, t)=2 k^{2} \operatorname{sech}^{2} k\left(x-4 k^{2} t-\eta_{0}\right)
$$

$$
\beta=4 k^{2}, \quad \xi=x, \quad \tau=t \quad \text { and } z_{0}=\eta_{0}
$$

In order to have a real solitary wave solution, the quantity $\beta$ must be a positive number. The wave moves to the right for $\beta>0$. The speed of propagation, denoted by $\beta$, is linearly related to the amplitude; therefore the amplitude is proportional to the speed. Thus larger amplitude waves move with a higher speed than smaller amplitude solitary waves.

\section{The Numerical Modelling of the One-Dimensional Form of the KdV Equation}

In the next two sections, various numerical methods are used to approximate the KdV equations. Taha and Ablowitz (1984) compared the efficiencies of different schemes ranging from finite difference methods for explicit and implicit methods to the study of finite fourier transform or pseudospectral methods. For this study, only explicit finite difference methods are studied. The aim here is to extend the valuable study of Taha and Ablowitz to compare several explicit methods. According to the approach given by the initial conditions, our main interest was to analyse which explicit scheme performs well when implemented to the KdV equation to produce the best soliton solution. Therefore, the following numerical methods are applied to the KdV equations (2.2) and (3.1):

1. First Order Upwind Scheme (Sweby, 2000);

2. Zabusky and Kruskal Scheme (Taha \& Ablowitz, 1984; Zabusky \& Kruskal, 1965); 
3. Lax-Wendroff Scheme (Sweby, 2000);

4. Walkley Scheme (Walkley, 1999);

In order to compare these schemes, equation (2.2) is used over a time interval beginning at $t=0$ and ending at $t$ $=T$ with initial conditions which match the analytic $1-$ Soliton and $2-$ Soliton solutions, the boundary conditions and the choice of parameter $\Delta x$ satisfying that of Taha and Ablowitz (1984).

\subsection{The Initial Conditions}

For a 1 - Soliton solution, the four explicit methods are applied to two different KdV equations using the following two different initial conditions.

$$
u_{t}+u u_{x}+\delta^{2} u_{x x x}=0
$$

where $\delta=0.022$, coupled with the initial conditions

$$
u(x, t)=\cos (\pi x), \quad 0 \leq x \leq 2
$$

where $u_{x}, u_{x x}, u_{x x x}$ periodic on $[0,2]$ for all $t$.

Zabusky and Kruskal have found the solution at the breakdown time of $t=\frac{1}{\pi}$ to have a slight oscillatory structure for $x<\frac{1}{2}$ due to the third derivative. The wave then steepens and almost produces a shock. At a later time of $t=\frac{3.6}{\pi}$, the solution shows a train of solitons, which have developed from the oscillations.

The Initial Condition for the $\operatorname{sech}^{2}$ Functionality

The exact 1 - Soliton solution of equation (2.2) on the infinite interval is

$$
u(x, t)=A \operatorname{sech}^{2}\left(k x-\omega t-\eta_{0}\right)
$$

where

$$
A=2 k^{2}, \omega=4 k^{3}, \quad k \text { and } \eta_{0} \text { are constants with } k>0
$$

This will represent a solitary wave of amplitude $2 k^{2}$ initially located at $x=-\frac{\eta_{0}}{k}$ and moving with velocity $4 k^{2}$. For initial conditions, equation (3.3) is used at $t=0$ and $\eta_{o}$ is chosen to be zero. For this initial condition, a single-soliton solution will be produced. The solitary wave will then move with a perfect advection along the $\mathrm{x}$-axis.

\subsection{The Separation into Two Solitons}

The exact 2 - Soliton solution of equation (2.2) on the infinite interval is

$$
u(x, t)=2 \frac{\partial^{2}}{\partial x^{2}}\left[\log _{e} f(x, t)\right]
$$

with

and

$$
\begin{aligned}
f(x, t) & =1+e^{\eta_{1}}+e^{\eta_{2}}+e^{\eta_{1}+\eta_{1}+A_{12}}, \\
\eta_{i} & =\eta_{i}(x, t)=k_{i} x-k_{i}^{3} t+\eta_{i}^{(0)},
\end{aligned}
$$

$$
e^{A_{i j}}=\left(\frac{k_{i}-k_{j}}{k_{i}+k_{j}}\right)^{2}
$$

where $k_{i}$ and $\eta_{i}^{(0)}$ are constants for $i=1,2 . k_{1}$ and $k_{2}$ are both taken to be positive. For $i=1,2$ soliton $i$ has an amplitude $\frac{k_{i}^{2}}{2}$ and moves with velocity $k_{i}^{2}$ from an initial location of $x=-\frac{\eta_{i}^{(0)}}{k_{i}}$.

By solving equation (3.4) using Mathematica, we obtain

$$
u(x, t)=\frac{2(a-c) e^{b+d-2\left(a^{3}+c^{3}\right) t+(a+c) x}\left(e^{\left(a^{3}+c^{3}\right) t}+e^{c x+d+a^{3} t}+e^{a x+b+c^{3} t}\right)}{\left(1+e^{a x+b-a^{3} t}+e^{c x+d-c^{3} t}+\frac{(a-c)^{2} e^{b+d-\left(a^{3}+c^{3}\right) t+(a+c) x}}{(a+c)^{2}}\right)^{2}}
$$

where

$$
a=k_{1}, \quad b=\eta_{1}^{(0)}, \quad c=k_{2} \text { and } \quad d=\eta_{2}^{(0)} .
$$


Therefore, this simplified version of the exact 2 - Soliton solution are used for the Fortran programming of all the explicit schemes of separation into two solitons.

For initial conditions, equation (3.4) is used at $t=0$, and two different sets of parameters are studied, i.e.

$$
\text { Parameter } 1 ; \quad k_{1}=1, k_{2}=\sqrt{2}, \eta_{1}^{(0)}=0, \eta_{2}^{(0)}=2 \sqrt{2}
$$

and

$$
\text { Parameter } 2 ; \quad k_{1}=1, k_{2}=\sqrt{5}, \eta_{1}^{(0)}=0, \eta_{2}^{(0)}=10.73
$$

The solution starts as an initial pulse, which then separates into two solitons each with different amplitudes. In time, the solitons will be allowed to interact and return to their original shapes.

\subsection{The Boundary Conditions}

For the initial condition of equation (3.1), Period Boundary Conditions are enforced on the interval $[0,2]$. To allow a numerical solution of equation (2.2), the 1 - Soliton solutions and 2 - Soliton solutions are assumed to satisfy the Periodic Boundary Conditions on the interval $[-20,20]$ i.e. $u(-20, t)=u(20, t)$.

\section{Finite Difference Methods}

To describe the schemes, the difference solution $u \equiv u(x, t)$ is specified numerically only at discrete points of $x=$ $n \Delta x, n=0,1, \ldots$ and $t=m \Delta t, m=0,1, \ldots$, where $\Delta x$ is the space step and $\Delta t$ is the time step.

All the schemes in Section 4.1 to 4.4 are explicit, which gives the solution at the time step explicitly in terms of the solution at earlier time steps.

\subsection{The First Order Upwind Scheme}

For the KdV equation (2.2) we first apply the First Order Upwind Scheme. With reference to Figure 4.1, linear advection stability is achieved for the upper scheme when the advection constant $v$ is negative and for the lower scheme it is stable when $v$ is positive. The Courant-Friedrichs-Lewy Stability criterion or simply Courant condition is satisfied here if $|v| \leq 1$. This scheme has several advantages over Centred schemes.

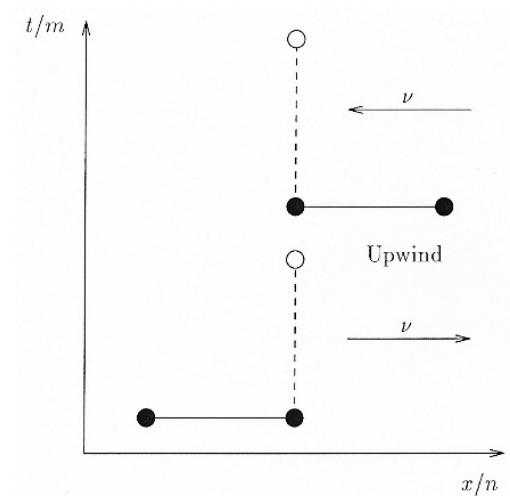

Figure 4.1. Representation of First order upwind schemes

For nonlinear advection upwind differencing, in general, adds stability to problems where the advected variables are vulnerable to sudden changes of state, such as passing through shock states or other discontinuities.

Conservative finite difference schemes for solving the Conservation Law, $u_{t}+f(u)_{x}=0$ where $f(u)=u$ can be written in the form

$$
u_{n}^{m+1}=u_{n}^{m}-\frac{\Delta t}{2 \Delta x}\left(h_{n+\frac{1}{2}}^{m}-h_{n-\frac{1}{2}}^{m}\right)
$$

where $h$ is a consistent numerical flux function and $u_{n}^{m} \approx u(n \Delta x, m \Delta t)$.

The First order upwind scheme may be written in this form as 


$$
\frac{u_{n}^{m+1}-u_{n}^{m}}{\Delta t}=-v_{n}^{m} \begin{cases}\frac{u_{n}^{m}-u_{n-1}^{m}}{\Delta x}, & v_{n}^{m}>0 \\ \frac{u_{n+1}^{m}-u_{n}^{m}}{\Delta t}, & v_{n}^{m}<0\end{cases}
$$

where the advection constant $v_{n}^{m}$ here is

$$
v_{n}^{m}=\frac{\Delta t}{\Delta x} \frac{f_{n+1}-f_{n}}{u_{n+1}-u_{n}}=\frac{\Delta t}{\Delta x}
$$

The advection part of the KdV equations (2.2) and (3.1) is used with

where

$$
h_{n+\frac{1}{2}}= \begin{cases}u_{n}, & \varphi_{n+\frac{1}{2}}>0 \\ u_{n+1,} & \varphi_{n+\frac{1}{2}}<0\end{cases}
$$

$$
\varphi_{n+\frac{1}{2}}=\frac{\Delta t}{\Delta x} \frac{f_{n+1}-f_{n}}{u_{n+1}-u_{n}}
$$

For the $\mathrm{KdV}$ equation in the form (3.1), where $f\left(u_{n}\right)=\frac{1}{2} u_{n}^{2}$, hence

$$
\varphi_{n+\frac{1}{2}}=\frac{\Delta t}{\Delta x} \frac{\left(u_{n+1}+u_{n}\right)}{2}
$$

and for the KdV equation (2.2) with $f\left(u_{n}\right)=3 u_{n}^{2}$

$$
\varphi_{n+\frac{1}{2}}=3 \frac{\Delta t}{\Delta x}\left(u_{n+1}+u_{n}\right)
$$

Applying equation (4.1) (where in this case $v_{n}^{m}=\varphi_{n+\frac{1}{2}}^{m}=\varphi_{n-\frac{1}{2}}^{m}$ ) to the KdV equation (2.2) i.e. $u_{t}+6 u u_{x}+$ $u_{x x x}=0$, together with a simple finite difference for the third derivative

$$
u_{n}^{m+1}=u_{n}^{m}-6 \frac{\Delta t}{\Delta x} u_{n}^{m}\left(h_{n+\frac{1}{2}}^{m}-h_{n-\frac{1}{2}}^{m}\right)-\frac{\Delta t}{2(\Delta x)^{3}}\left(u_{n+2}^{m}-2 u_{n+1}^{m}+2 u_{n-1}^{m}-u_{n-2}^{m}\right)
$$

and for the $\mathrm{KdV}$ equation (3.1),

$$
u_{n}^{m+1}=u_{n}^{m}-\frac{\Delta t}{\Delta x} u_{n}^{m}\left(h_{n+\frac{1}{2}}^{m}-h_{n-\frac{1}{2}}^{m}\right)-\frac{\delta^{2} \Delta t}{2(\Delta x)^{3}}\left(u_{n+2}^{m}-2 u_{n+1}^{m}+2 u_{n-1}^{m}-u_{n-2}^{m}\right)
$$

Following the conditions in (4.2), for $v_{n}^{m}>0$, equation (4.3) becomes

$$
u_{n}^{m+1}=u_{n}^{m}-6 \frac{\Delta t}{\Delta x} u_{n}^{m}\left(u_{n}^{m}-u_{n-1}^{m}\right)-\frac{\Delta t}{2(\Delta x)^{3}}\left(u_{n+2}^{m}-2 u_{n+1}^{m}+2 u_{n-1}^{m}-u_{n-2}^{m}\right)
$$

and for $v_{n}^{m}<0$, equation (4.3) becomes

$$
u_{n}^{m+1}=u_{n}^{m}-6 \frac{\Delta t}{\Delta x} u_{n}^{m}\left(u_{n+1}^{m}-u_{n}^{m}\right)-\frac{\Delta t}{2(\Delta x)^{3}}\left(u_{n+2}^{m}-2 u_{n+1}^{m}+2 u_{n-1}^{m}-u_{n-2}^{m}\right)
$$

The same format is achieved for equation (4.4).

This scheme is first order accurate in the calculation of the spatial derivatives. For both $v_{n}^{m}>0$ and $v_{n}^{m}<0$, the scheme has accuracy of order $(O(\Delta t)+O(\Delta x))$ and $\tau_{n}^{m} \rightarrow 0$ as $\Delta t \rightarrow 0, \Delta x \rightarrow 0$, hence the scheme is Consistent.

From the linearised form of the difference schemes equation (4.3) and equation (4.5) (with $u_{n}^{m}$ fixed as $\left.u_{0}\right)\left(v_{n}^{m}>0\right)$, we may carry out Fourier analysis for the stability to obtain

$$
\begin{aligned}
\xi & =1-\alpha\left[1-e^{i k \Delta x}\right]-\beta\left[e^{2 i k \Delta x}-2 e^{i k \Delta x}+2 e^{-i k \Delta x}-e^{-2 i k \Delta x}\right] \\
& =1-\alpha[1-\cos k \Delta x+i \sin k \Delta x]-\beta[4 i \sin k \Delta x(\cos k \Delta x-1)]
\end{aligned}
$$

where $\xi$ is the amplification factor. The first two terms lie on a circle with center $1-\alpha$ and radius $\alpha$ (see Figure 4.2 below) and $\xi$ is inside the unit circle if $\alpha \leq 1$. So, if $\beta=0$ it is conditionally stable. 


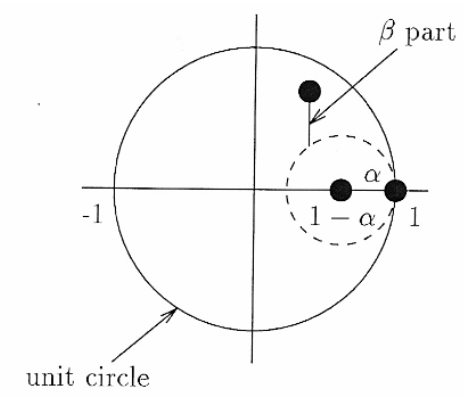

Figure 4.2. $\xi$ inside the unit circle for First Order Upwind Scheme

From equation (4.7)

$$
\begin{aligned}
|\xi|^{2}= & 1-\left[(1-\cos k \Delta x)\left(2 \alpha+2 \alpha^{2} \cos k \Delta x\right)\right] \\
& -\left(1-\cos ^{2} k \Delta x\right)\left[(\cos k \Delta x-1)\left(16 \beta^{2}(\cos k \Delta x-1)+8 \alpha \beta\right)\right]
\end{aligned}
$$

The stability criterion is $|\xi| \leq 1$ and is satisfied if $\alpha \leq 1$ and $\beta$ is taken to be sufficiently small.

To prove this stability criterion, we plot

$$
|\xi|^{2}=1-[(1-C) 2 \alpha(1+\alpha C)]-\left(1-C^{2}\right)\left[(C-1)\left(16 \beta^{2}(C-1)+8 \alpha \beta\right)\right] \leq 1
$$

for $\alpha, \beta$, and $\forall C$ using Matlab.

Equation (4.9) is a polynomial in $C,(C$ represents $\cos k \Delta x)$, i.e,

$$
\begin{aligned}
p(x)= & \left(16 \beta^{2}\right) C^{4}+\left(-32 \beta^{2}+8 \alpha \beta\right) C^{3}+\left(2 \alpha^{2}-8 \alpha \beta\right) C^{2} \\
& +\left(-2 \alpha^{2} 2 \alpha+32 \beta^{2}-8 \alpha \beta\right) C+\left(1-2 \alpha-16 \beta^{2}+8 \alpha \beta\right) .
\end{aligned}
$$

Therefore with Tables 1, 2, 3 and 4 representing Figures 4.3(a), (b), (c) and (d) respectively, when $\alpha<0,|\xi|^{2}>1$ (see Table 1), hence equation (4.7) will be unstable. Similar results are shown in Table 4, when $\alpha>1,|\xi|^{2}$ is also greater than 1. For equation (4.7) to satisfy the Fourier Stability Condition, $\alpha$ is taken to be between 0 and 1, i.e. $0 \leq \alpha \leq 1$ and $\beta$ is sufficiently small. This can be shown from Tables 2 and 3 , thus equation (4.7) is stable.

For equation (4.6), $\left(v_{n}^{m}<0\right)$, the amplification factor $\xi$ is

$$
\xi=1-\alpha[\cos k \Delta x+i \sin k \Delta x-1]-\beta[4 i \sin k \Delta x(\cos k \Delta x-1)]
$$

and also satisfies the Fourier Stability Condition, $|\xi|^{2} \leq 1$ for $-1 \leq \alpha \leq 0$, and $\beta$ taken to be sufficiently small.

Table 1. Values of $\alpha$ and $\beta$ for Figures 4.3(a)

\begin{tabular}{ccc}
\hline $\mathrm{W}$ & $\alpha$ & $\beta$ \\
\hline 1 & -1.5 & 0.1 \\
2 & -1.2 & 0.1 \\
3 & -1.0 & 0.1 \\
4 & -1.0 & 0.07 \\
5 & -0.5 & 0.05 \\
6 & -0.1 & 0.0 \\
\hline
\end{tabular}

Table 3. Values of $\alpha$ and $\beta$ for Figures 4.3(c)

\begin{tabular}{ccc}
\hline $\mathrm{y}$ & $\alpha$ & $\beta$ \\
\hline 1 & 0.6 & 0.0 \\
2 & 0.8 & 0.0 \\
3 & 0.9 & 0.0 \\
4 & 0.9 & 0.1 \\
5 & 1.0 & 0.0 \\
6 & 1.0 & 0.1 \\
\hline
\end{tabular}

Table 2. Values of $\alpha$ and $\beta$ for Figures 4.3(b)

\begin{tabular}{ccc}
\hline $\mathrm{X}$ & $A$ & $\beta$ \\
\hline 1 & 0.0 & 0.0 \\
2 & 0.05 & 0.05 \\
3 & 0.1 & 0.0 \\
4 & 0.2 & 0.0 \\
5 & 0.5 & 0.0 \\
6 & 0.5 & 0.1 \\
\hline
\end{tabular}

Table 4. Values of $\alpha$ and $\beta$ for Figures 4.3(d)

\begin{tabular}{ccc}
\hline $\mathrm{Z}$ & $\alpha$ & $\beta$ \\
\hline 1 & 1.05 & 0.1 \\
2 & 1.1 & 0.0 \\
3 & 1.1 & 0.1 \\
4 & 1.2 & 0.1 \\
5 & 1.5 & 0.05 \\
6 & 1.8 & 0.0 \\
\hline
\end{tabular}



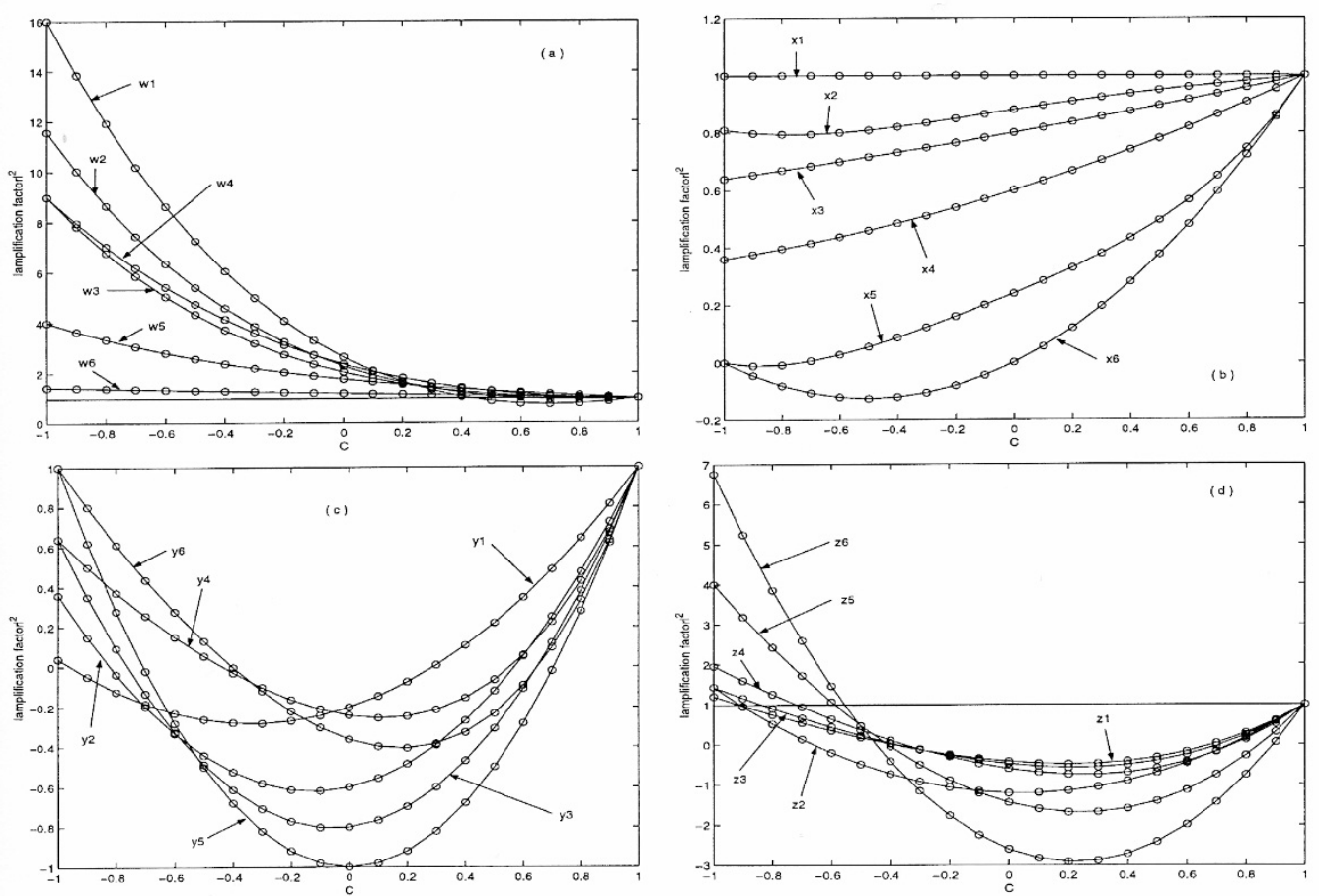

Figure 4.3. $|\xi|^{2} \leq 1$ against $C$ for stability criterion

The overall graphical results are not very good, the reason being the low order of the $1^{\text {st }}$ order scheme which is killing the accuracy of the high order $u_{x x x}$ approximation and the error is $O(\Delta x)$ in space.

In Figure 4.4, the First order upwind scheme is applied to the KdV equation (3.1), together with the initial condition of $u(x, t)=\cos (\pi x)$ on the interval $[0,2]$. The values of $\alpha$ and $\beta$ in this case are $u_{0} \frac{\Delta t}{\Delta x}$ and $\frac{\delta^{2} \Delta t}{2(\Delta x)^{3}}$

respectively. The wave profile at $t=\frac{1}{\pi}$ does not produce a shock but since this scheme is of low order, the profile did not reach $u(x, t)=-1$ as it should do in the other schemes. Another shock is also present when computed at time, $t=\frac{3.6}{\pi}$. Unfortunately here, there are no well-defined trains of eight solitons detected.

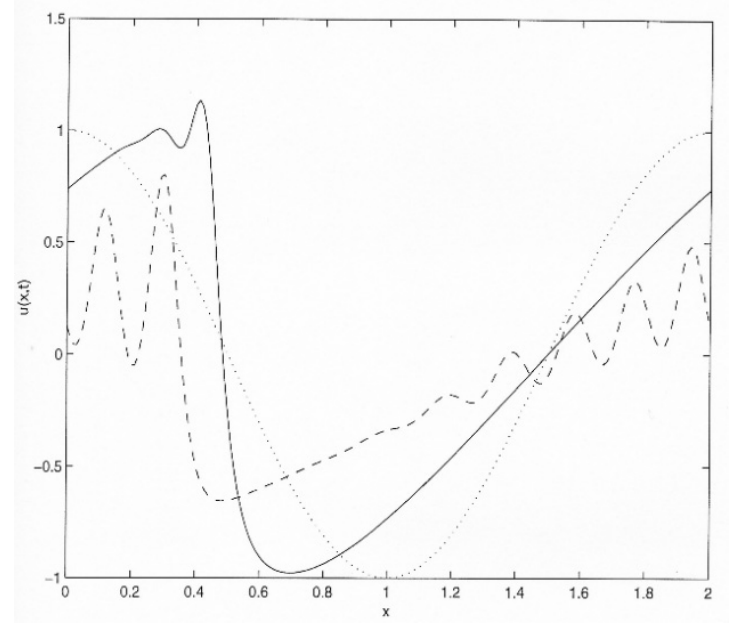

Figure 4.4. First Order Upwind Scheme as initial condition and $\delta=0.022$

Note: Initial profile at $t=0$ (indicated by the '.....' line); profile at $t=\frac{1}{\pi}$ (indicated by the '—— line); profile at $t=\frac{3.6}{\pi}$ (indicated by the ' - - - ' line); and $\Delta x=0.01, \Delta t=0.00001$ are used. 
Figures 4.5(a), (b) and (c) depicts the results of the second initial data for the $\operatorname{sech}^{2}$ function. The amplitudes decrease as time goes by. It is more obvious in Figure 4.5 (c) for amplitude $=4$. The scheme conserves mass but spreads.
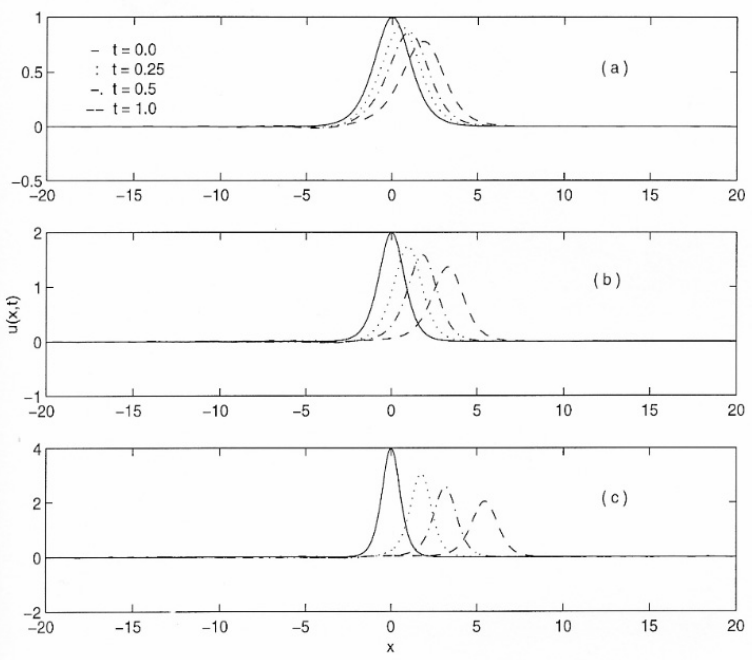

Figure 4.5. 1 - Soliton Solution for First order upwind scheme on the interval [-20, 20]

Note: Figure 4.5(a) amplitude $=1, \Delta x=0.1739, \Delta t=0.00001$; Figure 4.5(b) amplitude $=2, \Delta x=0.08, \Delta t=$ 0.000001 ; and Figure 4.5(c) amplitude $=4, \Delta x=0.05, \Delta t=0.0000001$.

The initial data for separation into two solitons (equation (3.4) is applied in Figure 4.6. In Figure 4.6(a) the two solitons took time to separate from each other and undoubtedly, the interaction of these two solitons may take longer to compute. It also seems that their amplitudes are almost similar. Poor results are expected in Figure 4.6(b). Two solitons of amplitudes $\frac{1}{2}$ and $\frac{5}{2}$ should be present instead three solitons, each with different amplitudes are produced. Hence, the First Order Upwind Scheme is a low order scheme that gives poor resolution of soliton effects.
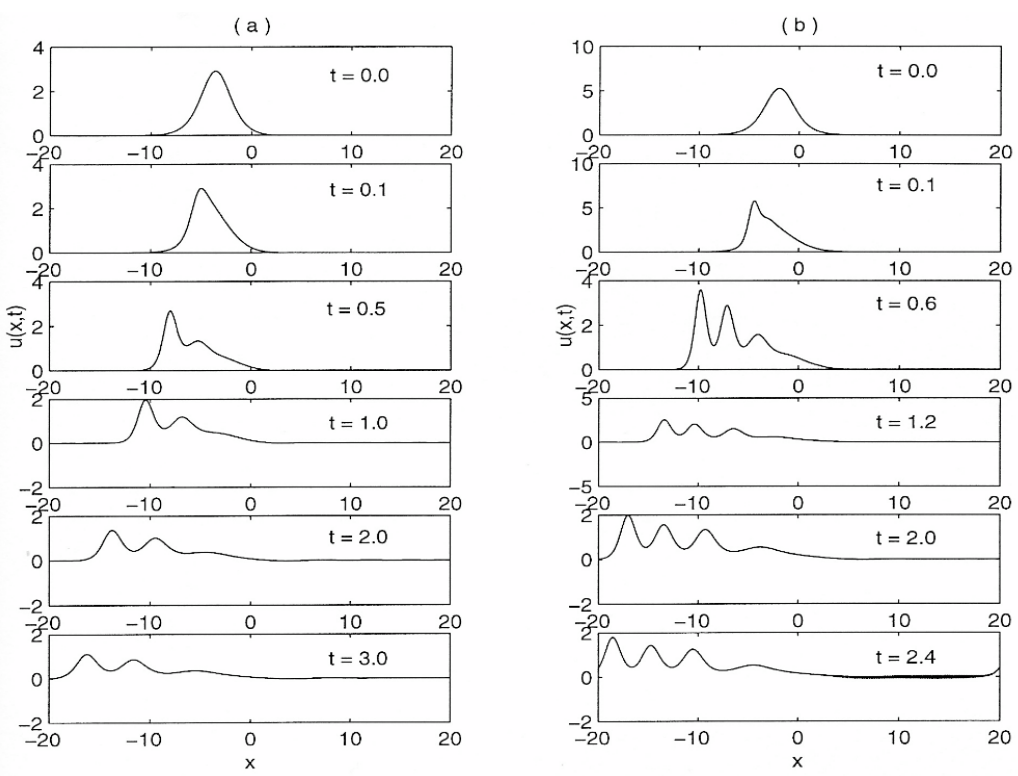

Figure 4.6. 2 - Soliton Solution for First order upwind Scheme on the interval [-20, 20]

Note: Figure 4.6(a) Parameter 1: amplitudes $=\frac{1}{2}$ and 1, $\Delta x=0.12, \Delta t=0.0000001$; and Figure 4.6(b)

Parameter 2: amplitudes $=\frac{1}{2}$ and $\frac{5}{2}, \Delta x=0.06, \Delta t=0.000001$. 


\subsection{The Zabusky and Kruskal Scheme}

Zabusky and Kruskal (1965) proposed the Explicit Leapfrog Finite Difference Scheme. They approximated equation (3.1) by

$$
\begin{gathered}
u_{n}^{m+1}=u_{n}^{m-1}-\frac{1}{3} \frac{\Delta t}{\Delta x}\left(u_{n+1}^{m}+u_{n}^{m}+u_{n-1}^{m}\right)\left(u_{n+1}^{m}-u_{n-1}^{m}\right) \\
-\frac{\delta^{2} \Delta t}{(\Delta x)^{3}}\left(u_{n+2}^{m}-2 u_{n+1}^{m}+2 u_{n-1}^{m}-u_{n-2}^{m}\right) \\
n=0,1, \ldots, N
\end{gathered}
$$

This scheme conserves 'mass' giving $\sum_{n=0}^{N-1} u_{n}^{m}$ independent of $n$.

Moreover, their choice of the average $\frac{1}{3}\left(u_{n+1}^{m}+u_{n}^{m}+u_{n-1}^{m}\right)$ to approximate $u$ in the nonlinear term $u_{x x}$ was to conserve the 'energy' to second order, since

$$
\frac{1}{2} \sum_{n=0}^{N-1}\left(u_{n}^{m+1}\right)^{2}-\frac{1}{2} \sum_{n=0}^{N-1}\left(u_{n}^{m-1}\right)^{2}=O\left((\Delta t)^{3}\right) \quad \text { as } \quad \Delta t \rightarrow 0
$$

if $u$ is periodic.

\section{Properties of the Scheme}

The Zabusky and Kruskal Scheme is a three-level scheme, which is a Second-order accurate in time. This scheme is Consistent ( $\tau_{n}^{m} \rightarrow 0$ as $\Delta t \rightarrow 0, \Delta x \rightarrow 0$ ) with equation (2.2) and the Truncation Error is of order $\left(\mathrm{O}\left((\Delta t)^{2}\right)+\mathrm{O}\left((\Delta x)^{2}\right)\right)$. The Linear Stability condition for this scheme is

$$
\frac{\Delta t}{\Delta x}\left|-2 u_{0}+\frac{1}{(\Delta x)^{2}}\right| \leq \frac{2}{3 \sqrt{3}}
$$

where $u_{0}$ is the maximum value of $u$ depending on the amplitude of solitons. To achieve this, a very small time step must be used in order to preserve stability.

\section{Application to the KdV Equation}

In the work of Taha and Ablowitz (1984), they have applied in the KdV equation (2.2) to the work of Zabusky and Kruskal (1965), so as to get the somewhat similar equation (4.10)

$$
\begin{gathered}
u_{n}^{m+1}=u_{n}^{m-1}-2 \frac{\Delta t}{\Delta x}\left(u_{n+1}^{m}+u_{n}^{m}+u_{n-1}^{m}\right)\left(u_{n+1}^{m}-u_{n-1}^{m}\right) \\
-\frac{\Delta t}{(\Delta x)^{3}}\left(u_{n+2}^{m}-2 u_{n+1}^{m}+2 u_{n-1}^{m}-u_{n-2}^{m}\right.
\end{gathered}
$$

where $u_{n}^{m}=u(n \Delta x, m \Delta t)$ also $n$ and $m$ are integers.

In order to evaluate the initial time step, the uncentred scheme

$$
\left.u_{n}^{1}=u_{n}^{0}-\frac{\Delta t}{\Delta x}\left(u_{n+1}^{0}+u_{n}^{0}+u_{n-1}^{0}\right)\left(u_{n+1}^{0}-u_{n-1}^{0}\right)-\frac{\Delta t}{2(\Delta x)^{3}}\left(u_{n+2}^{0}-2 u_{n+1}^{0}+2 u_{n-1}^{0}-u_{n-2}^{0}\right)\right)
$$

is applied.

The result of the Zabusky and Kruskal scheme is better than the First order upwind scheme, since the first moment is preserved.

We first present the results in Figure 4.7 with $u(x, t)=\cos (\pi x)$ as initial condition and $\delta=0.022$. The curve at $t$ $=0$ gives the initial wave profile while curve at $t=\frac{1}{\pi}$ shows the wave profile at the breakdown time which is equal to the reciprocal of absolute maximum of derivative of initial data. Here, the wave steepens and almost produces a shock, the slight oscillatory structure for $x<\frac{1}{2}$, in which case the third derivative, i.e. the dispersive term $\left(\delta^{2} u_{x x x}\right)$ then becomes significant. This is the result of local balance between nonlinearity and dispersion. At time $t=\frac{3.6}{\pi}$ the curve shows a train of eight well-defined waves which have developed since the early stage of evolution. Each of these eight solitons is like the $\operatorname{sech}^{2}$ functions, with the faster (taller) waves for ever 
catching-up and overtaking the slower (shorter) waves. To generate these wave profiles, very small time step of $\Delta t=0.0000002$ are used (hence longer runtime) in comparison to the other explicit schemes.

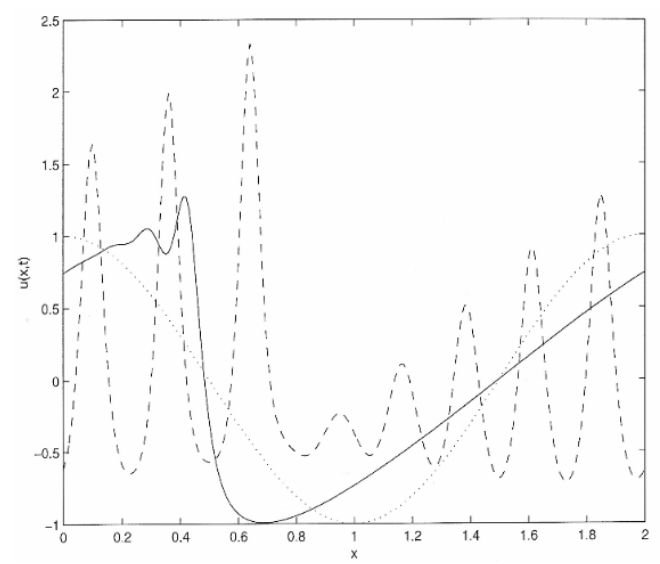

Figure 4.7. Zabusky and Kruskal Scheme using the Explicit Leapfrog Finite Difference Scheme as initial condition and $\delta=0.022$

Note: Initial profile at $t=0$ (indicated by the '.....' line); profile at $t=\frac{1}{\pi}$ (indicated by the '—— line); and profile at $t=\frac{3.6}{\pi}$ (indicated by the '- - -' line).

Equation (3.3), i.e. the initial condition for the $\operatorname{sech}^{2}$ function is applied in Figure 4.8. The Zabunsky and Kruskal scheme works perfectly here, as there is no spreading type of diffusion. For Figure 4.8(c), where it has a large amplitude of 4 , the solitary wave spreads out more because it is moving with a higher speed compared to the smaller amplitude solitary wave in Figures 4.8(a) and (b).
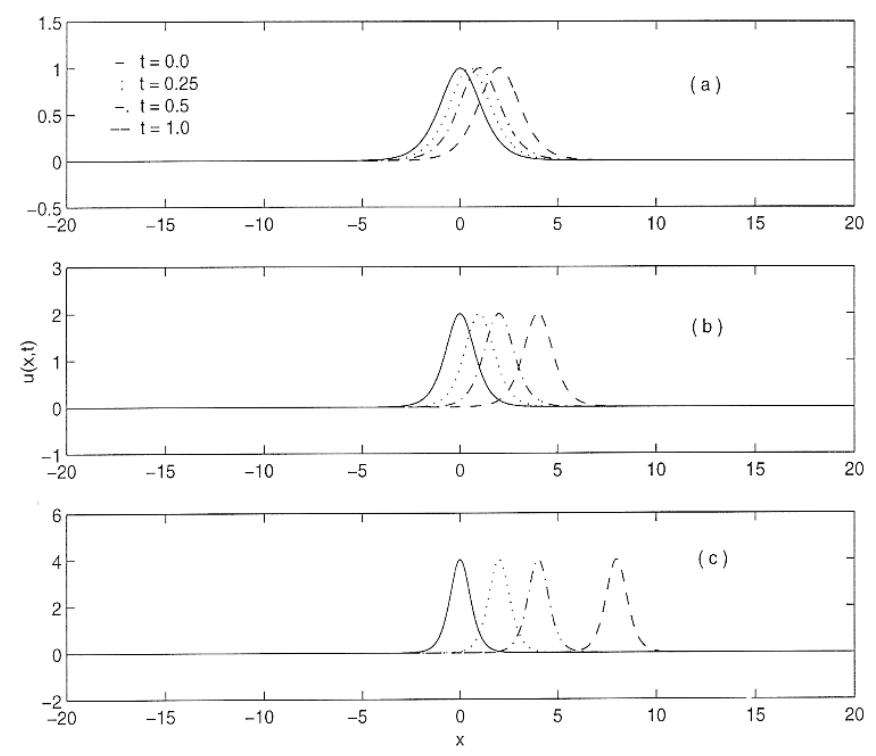

Figure 4.8. 1 - Soliton Solution for Zabusky and Kruskal Scheme on the interval [-20, 20]

Note: Figure 4.8(a) amplitude $=1, \Delta x=0.1739, \Delta t=0.002$; Figure 4.8(b) amplitude $=2, \Delta x=0.08, \Delta t=$ 0.00019 ; and Figure 4.8(c) amplitude $=4, \Delta x=0.05, \Delta t=0.00004$.

The third initial data, separation into two solitons, two different sets of parameters are studied, i.e. in Figure 4.9(a) and (b) for parameter 1 and 2 respectively. The solitons are run for times $t=0$ to $t=3$ for parameter 1 and $t=0$ to $t=2.4$ for parameter 2 , and after some time allowed to interact and return to their original shape. Unlike Figure 4.9(a), Figure 4.9(b) leaves blips in the middle, which is more visible than in (a). These blips are non-zero effect due to numerical errors. 

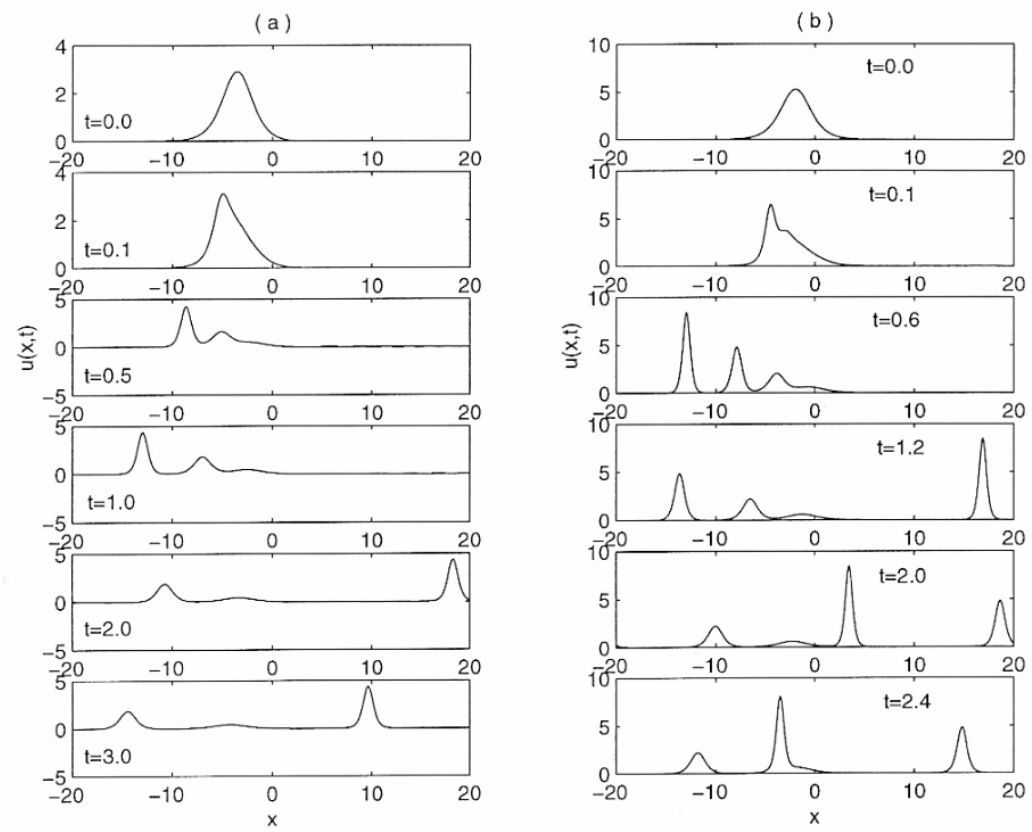

Figure 4.9. Separation into 2 - Solitons for Zabusky and Kruskal Scheme when they are allowed to separate on the interval $[-20,20]$

Note: Figure 4.9(a) Parameter 1: amplitudes $=\frac{1}{2}$ and 1, $\Delta x=0.12, \Delta t=0.00066$; and Figure 4.9(b) Parameter 2:

amplitudes $=\frac{1}{2}$ and $\frac{5}{2}, \Delta x=0.06, \Delta t=0.000082$.

\subsection{The Lax-Wendroff Scheme}

The next scheme to be applied to the KdV equation (2.2) is the Lax-Wendroff scheme, which is second-order in time and space. The Lax-Wendroff scheme is normally applied to the non-linear conservation law

The identity

$$
u_{t}+f_{x}=0
$$

is used, yielding

$$
u_{t t}=-\left(f_{x}\right)_{t}=-\left(f_{t}\right)_{x}=-\left(f^{\prime}(u) u_{t}\right)_{x}=\left(f^{\prime}(u) f_{x}\right)_{x}
$$

$$
u_{n}^{m+1}=u_{n}^{m}-\frac{1}{2} \frac{\Delta t}{\Delta x}\left(f_{n+1}^{m}+f_{n-1}^{m}\right)+\frac{1}{2} \frac{\Delta t}{\Delta x}\left[\varphi_{n+\frac{1}{2}}\left(f_{n+1}^{m}-f_{n}^{m}\right)-\varphi_{n-\frac{1}{2}}\left(f_{n}^{m}-f_{n-1}^{m}\right)\right]
$$

as the Lax-Wendroff scheme for the non-linear conservation law.

As in equation (4.1) the conservation form is

$$
u_{n}^{m+1}=u_{n}^{m}-\frac{1}{2} \frac{\Delta t}{\Delta x}\left(h_{n+\frac{1}{2}}^{m}+h_{n-\frac{1}{2}}^{m}\right)
$$

where

$$
h_{n+\frac{1}{2}}^{m}=h\left(u_{n-l}^{m}, \ldots, u_{n+r}^{m}\right)
$$

with $h$ a consistent numerical flux functionality

$$
h(u, \ldots, u)=f(u)
$$

Properties of the Scheme

The One-step Lax-Wendroff differencing scheme applied to advection is second order accurate in time and space. Its order of accuracy is $\left(\mathrm{O}\left((\Delta t)^{2}\right)+\mathrm{O}\left((\Delta x)^{2}\right)\right)$ and hence Consistency is achieved for Truncation error $\tau_{n}^{m} \rightarrow 0$ as $\Delta t \rightarrow 0, \Delta x \rightarrow 0$. 
Investigating the linear stability of this scheme for the $\mathrm{KdV}$ equation (2.2) with a simple difference for the $u_{x x x}$ term and $f=6 u_{0} u$ gives

$$
\begin{aligned}
\xi^{m+1} e^{i n k \Delta x}=\xi^{m} e^{i n k \Delta x}-\left[\alpha\left(\xi^{m} e^{i(n+1) k \Delta x}-\xi^{m} e^{i(n-1) k \Delta x}\right)-2 \alpha\left(\xi^{m} e^{i(n+1) k \Delta x}+\xi^{m} e^{i(n-1) k \Delta x}\right)\right. & \\
& \left.+4 \alpha \xi^{m} e^{i n k \Delta x}\right]-\beta\left[\xi^{m} e^{i(n+2) k \Delta x}-\xi^{m} e^{i(n+1) k \Delta x}-2 \xi^{m} e^{i(n-1) k \Delta x}-\xi^{m} e^{i(n-2) k \Delta x}\right.
\end{aligned}
$$

where $\alpha$ here is $3 u_{0} \frac{\Delta t}{\Delta x}, \beta=\frac{\Delta t}{2(\Delta x)^{3}}$ and $\xi$ is the amplification factor.

$$
\begin{aligned}
\xi & =1-\alpha\left[\left(e^{i k \Delta x}-e^{-i k \Delta x}\right)-2 \alpha\left(e^{i k \Delta x}+e^{-i k \Delta x}\right)+4 \alpha\right] \\
& \quad-\beta\left[e^{2 i k \Delta x}-2 e^{i k \Delta x}+2 e^{-i k \Delta x}-e^{-2 i k \Delta x}\right] \\
& =1-\alpha[2 i \sin k \Delta x-2 \alpha(\cos k \Delta x)+4 \alpha]+\beta[4 i \sin k \Delta x(1-\cos k \Delta x)] \\
& =1-4 \alpha^{2}(1-\cos k \Delta x)-2 \alpha i \sin k \Delta x+\beta[4 i \sin k \Delta x(1-\cos k \Delta x)]
\end{aligned}
$$

With reference to Figure 4.10 , the $\xi$ lies on an ellipse, centre $\left(1-4 \alpha^{2}, 0\right)$ with semi-axes $\left(4 \alpha^{2},-4 \alpha\right) . \xi$ is inside the unit circle if $\left|4 \alpha^{2}\right|<1$ then the scheme is conditionally stable.

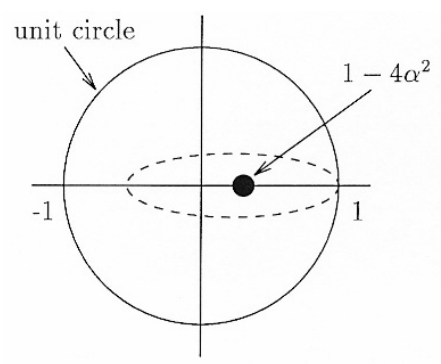

Figure 4.10. $\xi$ inside the unit circle for Lax-Wendroff scheme

For the Fourier Stability Criterion $\left|\xi^{2}\right| \leq 1$, we require $\left|4 \alpha^{2}\right| \leq 1$ and $\beta$ has to be sufficiently small. Thus,

$$
\begin{aligned}
|\xi|^{2}=1 & -\left[8 \alpha^{2}(1-\cos k \Delta x)\right]+16 \alpha^{4}(1-\cos k \Delta x)^{2}-\left(1-\cos ^{2} k \Delta x\right)\left[4 \alpha^{2}\right. \\
& \left.+16 \beta^{2}(1-\cos k \Delta x)^{2}-16 \alpha \beta(1-\cos k \Delta x)\right]
\end{aligned}
$$

To check that $|\xi|^{2} \leq 1 \forall \alpha, \beta$ and $\cos k \Delta x \equiv C$, we use equation (4.16) and transforming it into equation (4.17) below to get

$$
\begin{aligned}
p(x)= & \left(16 \beta^{2}\right) C^{4}+\left(-32 \beta^{2}+16 \alpha \beta\right) C^{3}+\left(16 \alpha^{4}+4 \alpha^{2}-16 \alpha \beta\right) C^{3} \\
& +\left(-32 \alpha^{4}+8 \alpha^{2}+32 \beta^{2}-16 \alpha \beta\right) C+\left(1+16 \alpha^{4}-12 \alpha^{2}-16 \beta^{2}+16 \alpha \beta\right.
\end{aligned}
$$

Equation (4.16) is a polynomial in C. In Matlab we plot equation (4.17) hence Figures 4.11(a) and (b) are produces with their values of $\alpha+\beta$ presented in Tables 5 and 6. In order to satisfy the Fourier Stability Condition for equation (4.15), $-0.5 \leq \alpha \leq 0.5$ as shown for $\mathrm{x} 3 \rightarrow \mathrm{x} 8$ and $\mathrm{y} 1 \rightarrow \mathrm{y} 6$ in Figures 4.11(a) and (b) respectively. Also $\beta$ has to be sufficiently small so, $|\xi|^{2} \leq 1$.
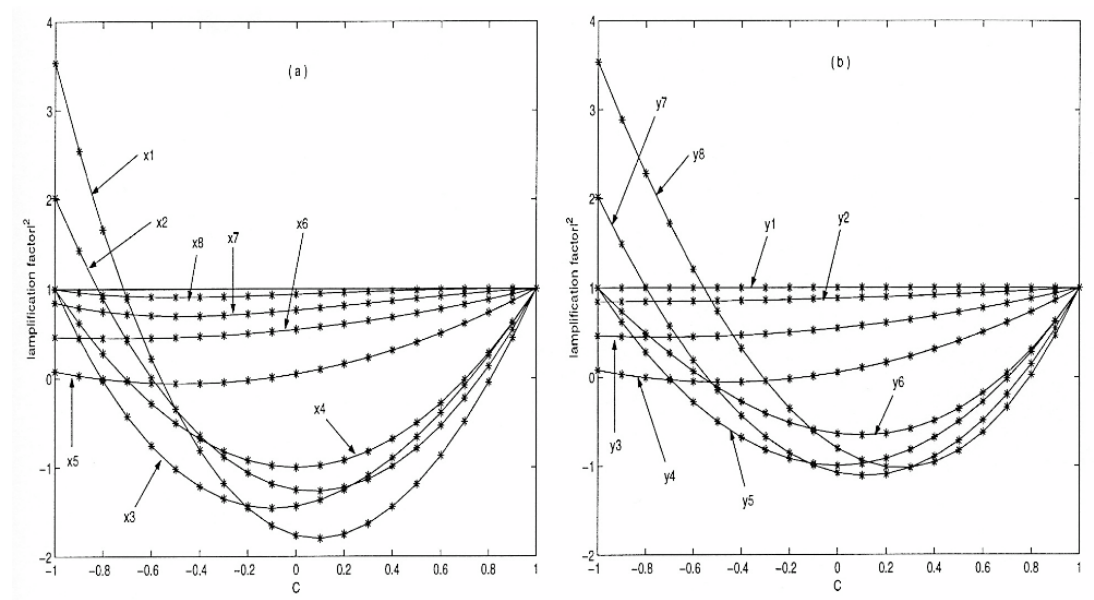

Figure 4.11. $|\xi|^{2} \leq 1$ against $C$ for stability criterion 
Table 5. Values for $\alpha+\beta$ for Figures 4.11(a)

\begin{tabular}{ccc}
\hline $\mathrm{x}$ & $A$ & $\beta$ \\
\hline 1 & -0.6 & 0.05 \\
2 & -0.55 & 0.01 \\
3 & -0.5 & 0.05 \\
4 & -0.5 & 0.0 \\
5 & -0.3 & 0.0 \\
6 & -0.2 & 0.0 \\
7 & -0.1 & 0.05 \\
8 & -0.02 & 0.05 \\
\hline
\end{tabular}

Table 6. Values for $\alpha+\beta$ for Figures 4.11(b)

\begin{tabular}{ccc}
\hline$\alpha$ & $\beta$ & $\alpha$ \\
\hline 0.0 & 0.0 & 0.0 \\
0.1 & 0.0 & 0.1 \\
0.2 & 0.0 & 0.2 \\
0.3 & 0.0 & 0.3 \\
0.5 & 0.0 & 0.5 \\
0.5 & 0.05 & 0.5 \\
0.55 & 0.01 & 0.55 \\
0.6 & 0.05 & 0.6 \\
\hline
\end{tabular}

\section{Application to the KdV Equation}

Equation (4.1) is used for applying to the advection part of the KdV equation (3.1) where $f\left(u_{n}\right)=\frac{1}{2} u_{n}^{2}$, hence

$$
h_{n+\frac{1}{2}}=\frac{1}{2}\left(\left(u_{n+1}+u_{n}\right)-\varphi_{n+\frac{1}{2}}\left(u_{n+1}-u_{n}\right)\right)
$$

and

$$
\varphi_{n+\frac{1}{2}}=\frac{\Delta t}{\Delta x} \frac{\left(f_{n+1}-f_{n}\right)}{\left(u_{n+1}-u_{n}\right)}=\frac{\Delta t}{\Delta x} \frac{\left(u_{n+1}+u_{n}\right)}{2}
$$

Therefore

$$
h_{n-\frac{1}{2}}=\frac{1}{2}\left(\left(u_{n}+u_{n-1}\right)-\varphi_{n-\frac{1}{2}}\left(u_{n}-u_{n-1}\right)\right)
$$

and

$$
\varphi_{n-\frac{1}{2}}=\frac{\Delta t}{\Delta x} \frac{\left(u_{n}+u_{n-1}\right)}{2}
$$

The scheme for equation (3.1) will therefore become

$$
\begin{aligned}
u_{n}^{m+1}= & u_{n}^{m}-\frac{\Delta t}{2 \Delta x} u_{n}^{m}\left[\left(u_{n+1}^{m}+u_{n}^{m}\right)-\frac{\Delta t}{2 \Delta x}\left(\left(u_{n+1}^{m}\right)^{2}-\left(u_{n}^{m}\right)^{2}\right)-\left(u_{n}^{m}+u_{n-1}^{m}\right)\right. \\
& \left.+\frac{\Delta t}{2 \Delta x}\left(\left(u_{n}^{m}\right)^{2}-\left(u_{n-1}^{m}\right)^{2}\right)\right]-\frac{\delta^{2} \Delta t}{(\Delta x)^{3}}\left(u_{n+2}^{m}-2 u_{n+1}^{m}+2 u_{n-1}^{m}-u_{n-2}^{m}\right)
\end{aligned}
$$

For equation (2.2) (where $\left.f\left(u_{n}\right)=3 u_{n}^{2}\right)$ with $\varphi_{n+\frac{1}{2}}=3 \frac{\Delta t}{\Delta x}\left(u_{n+1}+u_{n}\right), \varphi_{n-\frac{1}{2}}=3 \frac{\Delta t}{\Delta x}\left(u_{n}+u_{n-1}\right)$ and using again a simple difference for the third order term, hence the scheme will be

$$
\begin{aligned}
u_{n}^{m+1}= & u_{n}^{m}-3 \frac{\Delta t}{\Delta x} u_{n}^{m}\left[\left(u_{n+1}^{m}+u_{n}^{m}\right)-3 \frac{\Delta t}{\Delta x}\left(\left(u_{n+1}^{m}\right)^{2}+\left(u_{n}^{m}\right)^{2}\right)-\left(u_{n}^{m}+u_{n-1}^{m}\right)\right. \\
& \left.+3 \frac{\Delta t}{\Delta x}\left(\left(u_{n}^{m}\right)^{2}-\left(u_{n-1}^{m}\right)^{2}\right)\right]-\frac{\Delta t}{2(\Delta x)^{3}}\left(u_{n+2}^{m}-2 u_{n+1}^{m}+2 u_{n-1}^{m}-u_{n-2}^{m}\right)
\end{aligned}
$$

The Lax-Wendroff scheme produces good results and the scheme works well. The initial condition for the cos function and $\delta=0.022$ are applied in Figure 4.12. 


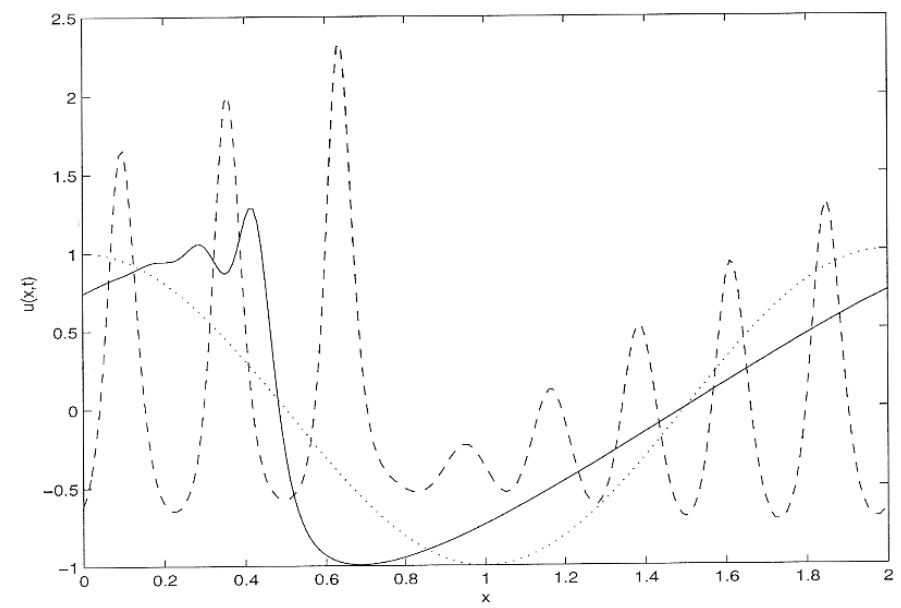

Figure 4.12. Lax-Wendroff Scheme as initial condition and $\delta=0.022$.

Note: Initial profile at $t=0$ (indicated by the ' ....' line); profile at $t=\frac{1}{\pi}$ (indicated by the '—— line); and profile at $t=\frac{3.6}{\pi}$ (indicated by the '- - -' line).

The curves in Figure 4.12 are similar to the ones for the Zabusky and Kruskal scheme where the wave profile at $t=\frac{1}{\pi}$ shows the slight shock for $x>\frac{1}{2}$. The train of solitons are also present here for $t=\frac{3.6}{\pi}$. For this figure, the Lax-Wendroff scheme does takes shorter runtime than the Zabusky and Kruskal scheme, but longer runtime hence more computing time for the other two figures (4.13 and 4.14).

The results of the initial data of the 1 - Soliton solution for the $\operatorname{sech}^{2}$ function are shown in Figures 4.13(a), (b) and (c). Here, the solitons for time $t=0.25,0.5$ and 1.0 with their respective amplitudes moves constantly on the interval $[-20,20]$. As the amplitudes get bigger, the solitons spread out more (shown in Figure 4.13(c)).
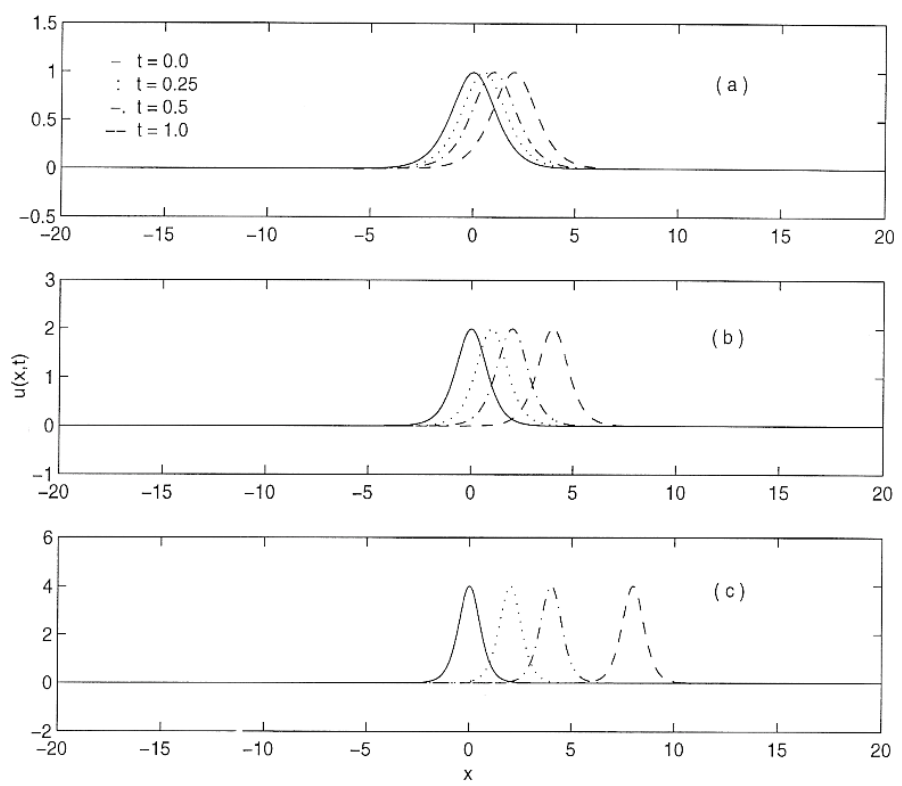

Figure 4.13. 1 - Soliton Solution for Lax-Wendroff Scheme on the interval [-20, 20].

Note: Figure 4.13(a) amplitude $=1, \Delta x=0.1739, \Delta t=0.00001$; Figure 4.13(b) amplitude $=2, \Delta x=0.08, \Delta t=$ 0.000001 ; and Figure 4.13(c) amplitude $=4, \Delta x=0.05, \Delta t=0.0000001$.

Again, similar results like that of Figure 4.9(a) are produced for Figure 4.14(a) when the initial conditions for the 2 - Soliton test problem with Parameter 1 are applied. In Figure 4.14(b), the two solitons with amplitudes $\frac{1}{2}$ and $\frac{5}{2}$ separates after $t=0.1$, moves apart from each other and they will interact in time but yet still preserve their 
shapes. At $t=0.6$, Figure 4.14(b) starts to leave humps in the middle (non-zero effect due to numerical errors) and these humps are less noticeable in Figure 4.14(a). Unfortunately, at $t=2.4$ the oscillations are a bit obvious, so a smaller $\Delta t$ should be used.
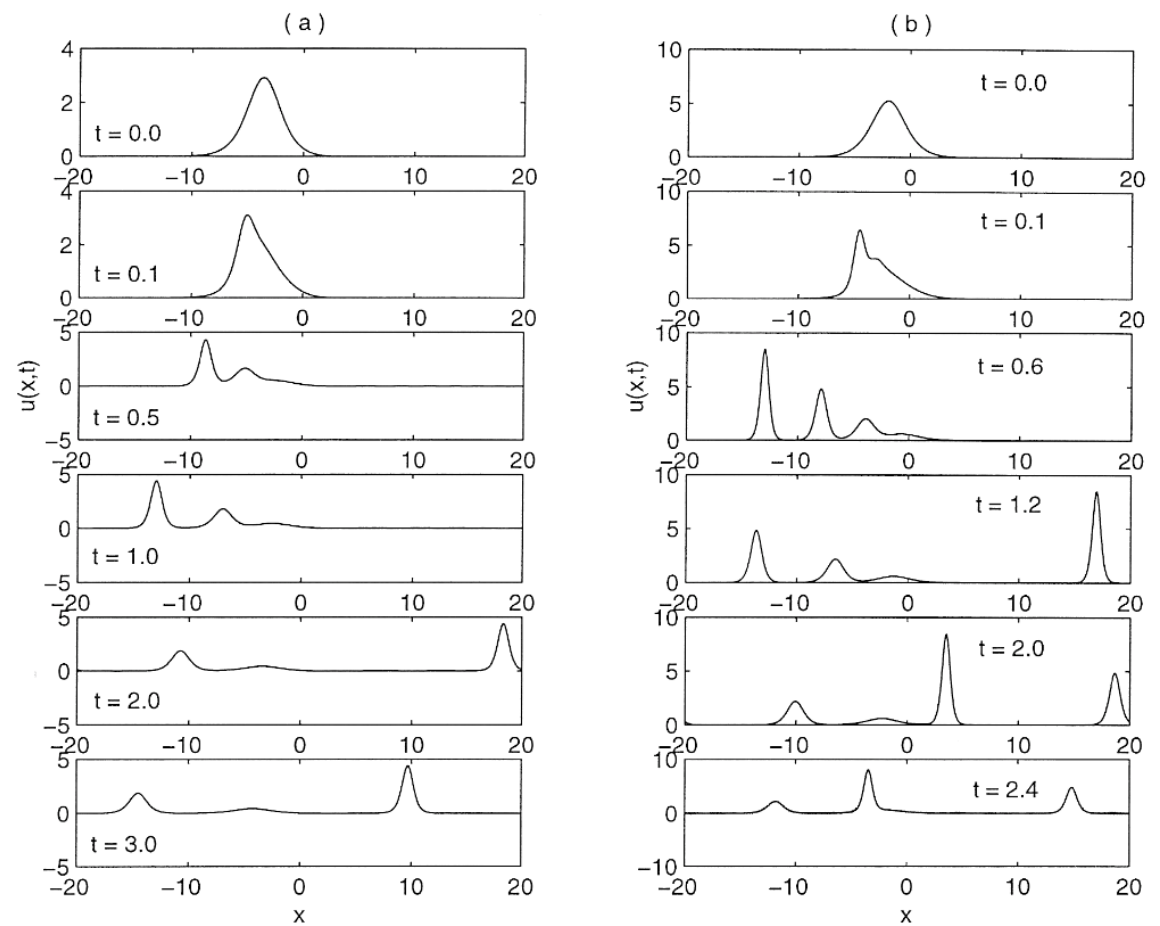

Figure 4.14. Separation into Two Soliton for Lax-Wendroff Scheme on the interval [-20, 20]

Note: Figure 4.14(a) Parameter 1: amplitudes $=\frac{1}{2}$ and 1, $\Delta x=0.12, \Delta t=0.0000001$; and Figure 4.14(b) Parameter 2: amplitudes $=\frac{1}{2}$ and $\frac{5}{2}, \Delta x=0.06, \Delta t=0.0000001$.

\subsection{The Walkley Scheme}

Walkley (1999) has applied a new scheme to the KdV equation (2.2) in connection with modelling a Boussinesq equation for water flows. In dimensional variables, it is given as

$$
\frac{\delta u}{\delta t}+\sqrt{g H} \frac{\delta u}{\delta x}+\frac{3}{2} u \frac{\delta u}{\delta x}+\frac{H^{2}}{6} \sqrt{g H} \frac{\delta^{3} u}{\delta t^{3}}=0
$$

( $H$ here is the depth of water), to investigate numerical solution procedures for dispersive wave equations. With reference to equation (2.2), he has illustrated a new approach to finite difference methods used in the spatial approximation of the equation.

\section{Spatial Discretisation}

The $\mathrm{KdV}$ equation (4.20) is rewritten in an easy form before being applied for use in the spatial approximations

$$
\dot{u}=-\frac{\delta f}{\delta x}-\varsigma \frac{\delta^{3} u}{\delta x^{3}}
$$

where

$$
\dot{u}=-\frac{\delta u}{\delta t} \quad \text { and } \quad f=a u+\frac{b}{2} u^{2} .
$$

The problem domain is $x \in\left[X_{1}, X_{2}\right]$ and $t \in[0, T]$. The three sets of initial conditions for the time integration are evaluated at $t=0$.

Outline of the Basic Methodologies 
As usual the spatial domain of the problem $x \in\left[X_{1}, X_{2}\right]$ is covered with a set of $N+1$ equally spaced points $\left\{x_{1}, x_{2}, \ldots, x_{N}, x_{N+1}\right\}$, where

$$
\begin{gathered}
x_{n}=X_{1}+(n-1) \Delta x, \quad 1 \leq n \leq N+1 \\
\Delta x=\frac{x_{2}-x_{1}}{N}
\end{gathered}
$$

Spatial derivatives are then approximated directly at a given point by using Taylor series expansions in space in order to relate the derivative at that point to adjacent nodal solution values. This leads to a global equation system with a banded matrix structure.

Equation (4.21) contains first, second and third spatial derivatives. With respect to the space step, finite difference approximations of second and fourth order accuracy are shown in the following equations below and for accuracy of these expressions the leading truncation error terms from the Taylor series approximations are also included.

For the first spatial derivatives

$$
\begin{aligned}
& \frac{d f}{d x}\left(x_{n}\right)=\frac{1}{2 \Delta x}\left(f_{n+1}-f_{n-1}\right)-\frac{1}{6}(\Delta x)^{2} \frac{d^{3} f}{d x^{3}}\left(x_{n}\right)+O\left((\Delta x)^{4}\right) \\
& \frac{d f}{d x}\left(x_{n}\right)=\frac{1}{12 \Delta x}\left(f_{n-2}-8 f_{n-1}+8 f_{n+1}-f_{n+2}\right)-\frac{1}{90}(\Delta x)^{4} \frac{d^{5} f}{d x^{5}}\left(x_{n}\right)+O\left((\Delta x)^{6}\right)
\end{aligned}
$$

For the second spatial derivatives

$$
\begin{aligned}
\frac{d^{2} f}{d x^{2}}\left(x_{n}\right)= & \frac{1}{(\Delta x)^{2}}\left(f_{n-2}-2 f_{n}+f_{n-1}\right)-\frac{1}{12}(\Delta x)^{2} \frac{d^{4} f}{d x^{4}}\left(x_{n}\right)+O\left((\Delta x)^{4}\right) \\
\frac{d^{2} f}{d x^{2}}\left(x_{n}\right)= & \frac{1}{12(\Delta x)^{2}}\left(-f_{n-2}+16 f_{n-1}-30 f_{n}+16 f_{n-1}-f_{n-2}\right) \\
& \quad+\frac{1}{90}(\Delta x)^{4} \frac{d^{6} f}{d x^{6}}\left(x_{n}\right)+O\left((\Delta x)^{6}\right)
\end{aligned}
$$

For the third spatial derivatives

$$
\begin{gathered}
\frac{d^{3} f}{d x^{3}}\left(x_{n}\right)=\frac{1}{2(\Delta x)^{3}}\left(-f_{n-2}+2 f_{n-1}-2 f_{n+1}+f_{n+2}\right)-\frac{7}{60}(\Delta x)^{2} \frac{d^{5} f}{d x^{5}}\left(x_{n}\right)+O\left((\Delta x)^{4}\right) \\
\frac{d^{3} f}{d x^{3}}\left(x_{n}\right)=\frac{1}{8(\Delta x)^{3}}\left(f_{n-3}-8 f_{n-2}+13 f_{n-1}-13 f_{n+1}+8 f_{n+2}-f_{n+3}\right) \\
+\frac{7}{120}(\Delta x)^{4} \frac{d^{7} f}{d x^{7}}\left(x_{n}\right)+O\left((\Delta x)^{6}\right)
\end{gathered}
$$

All the difference stencils shown above are centred on the node $n$.

\section{Finite Difference Methods for the KdV Equation}

It is argued that a Second order finite difference method, using stencils (4.22) and (4.26) will introduce a third spatial derivative where the leading truncation error term is from the first derivative of (4.22). If it is used in the $\mathrm{KdV}$ equation (4.21) this will automatically put in an additional numerical dispersion of the same type as appeared in the system originally and is likely to perform badly in numerical tests.

A fully Fourth order finite difference method, using stencils (4.23) and (4.27) however will produce a scheme of at least fifth order derivatives in the truncation error. The KdV equation (4.21) will have a sufficient accuracy but at the cost of a larger support over nodes $n ; n-3$ to $n+3$. By replacing the first derivative with the fourth order approximation (4.23), and the third derivative with the second order approximation (4.26), this scheme will be sufficiently accurate and have a smaller support over nodes $n ; n-2$ to $n+2$.

For the KdV equation (4.21) the finite difference scheme with the leading truncation error terms at a node $n$ is

$$
\begin{gathered}
\dot{u}_{n}=-\frac{1}{12 \Delta x}\left(f_{n-2}-8 f_{n-1}+8 f_{n+1}-f_{n+2}\right)-\frac{\varsigma}{(2 \Delta x)^{3}}\left(u_{n+2}-2 u_{n+1}+2 u_{n-1}-u_{n-2}\right) \\
-\frac{7 \varsigma}{60}(\Delta x)^{2} \frac{d^{5} f}{d x^{5}}\left(x_{n}\right)+\frac{1}{90}(\Delta x)^{4} \frac{d^{5} f}{d x^{5}}\left(x_{n}\right)
\end{gathered}
$$


This scheme is only second-order accurate but should not produce significant numerical dispersion.

To apply equation (4.28) into the $\mathrm{KdV}$ equation (2.2), for $f=a u+\frac{b}{2} u^{2}, a$ is taken to be 0 and $b$ is 6 , therefore $f=3 u^{2}$. With substitution of $f=3 u^{2}, \varsigma=1, \dot{u}_{n}=\frac{u_{n}^{m+1}-u_{n}^{m}}{\Delta t}$ and excluding the leading truncation error terms, equation (4.28) becomes

$$
\begin{aligned}
u_{n}^{m+1}= & u_{n}^{m}-\frac{1}{12} \frac{\Delta t}{\Delta x}\left[3\left(u_{n-2}^{m}\right)^{2}-8\left(3\left(u_{n-1}^{m}\right)^{2}\right)+8\left(3\left(u_{n+1}^{m}\right)^{2}-3\left(u_{n+2}^{m}\right)^{2}\right]\right. \\
& -\frac{\Delta t}{(2 \Delta x)^{3}}\left(u_{n+2}^{m}-2 u_{n+1}^{m}+2 u_{n-1}^{m}-u_{n-2}^{m}\right)
\end{aligned}
$$

For the KdV equation (3.1) where $a=0$ and $b=1$, hence $f=\frac{1}{2} u^{2}$ and $\varsigma=1$, equation (4.28) will be

$$
\begin{aligned}
u_{n}^{m+1}= & u_{n}^{m}-\frac{1}{12} \frac{\Delta t}{\Delta x}\left[\frac{1}{2}\left(u_{n-2}^{m}\right)^{2}-4\left(u_{n-1}^{m}\right)^{2}+4\left(u_{n+1}^{m}\right)^{2}-\frac{1}{2}\left(u_{n+2}^{m}\right)^{2}\right] \\
& -\frac{\delta^{2} \Delta t}{(2 \Delta x)^{3}}\left(u_{n+2}^{m}-2 u_{n+1}^{m}+2 u_{n-1}^{m}-u_{n-2}^{m}\right)
\end{aligned}
$$

The Walkley scheme had a $3^{\text {rd }}$ order $u u_{x x}$ approximation to avoid polluting the dispersive term $u u_{x x x}$ approximation. It is first-order in time. For Fourier stability using the linearised equation, it has the amplification factor of

$$
\xi=1-\alpha\left(3 i \sin k \Delta x+2 i \sin k \Delta x\left(\sin ^{2} \frac{1}{2} k \Delta x\right)\right)+\beta\left(2 i \sin k \Delta x\left(\sin ^{2} \frac{1}{2} k \Delta x\right)\right)
$$

where $\alpha=2 u_{0} \frac{\Delta t}{\Delta x}$ and $\beta=2 \frac{\Delta t}{(\Delta x)^{3}}$.

$\xi$ is always outside the unit circle because all terms except the first are imaginary, so absolutely unstable $\forall k$.

We have come to a conclusion that, even though the Walkley scheme is linearly Fourier unstable, since the KdV equation applied to this scheme is nonlinear in nature therefore the nonlinearity keeps them stable. Linear stability theory is therefore unreliable.

The Walkley scheme works well, just like that of the Zabusky and Kruskal scheme and also the Lax-Wendroff scheme. It produces the same curves for Figure 4.15 with the first set of initial condition i.e. $u(x, t)=\cos (\pi x)$.

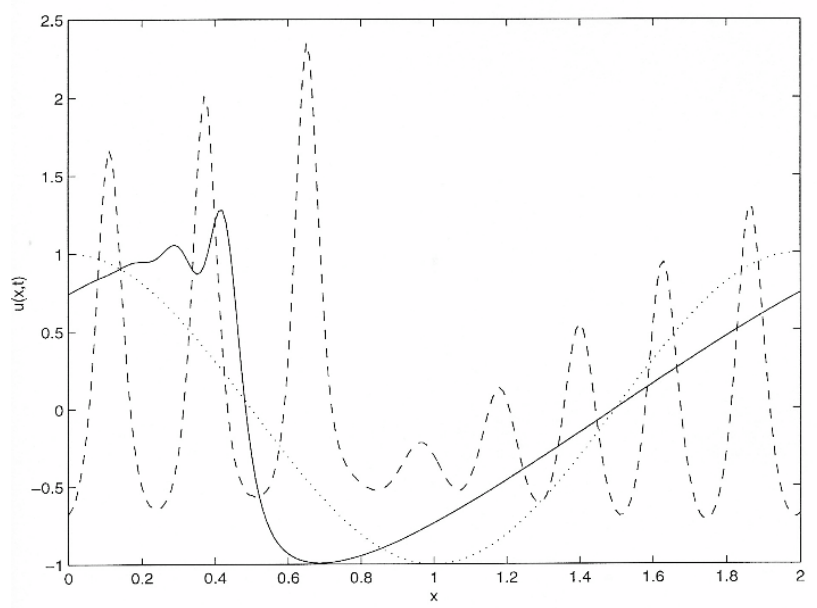

Figure 4.15. Walkley Scheme as initial condition and $\delta=0.022$

Note: Initial profile at $t=0$ (indicated by the '.....' line); profile at $t=\frac{1}{\pi}$ (indicated by the '- ' line); profile at $t=\frac{3.6}{\pi}$ (indicated by the '- - -' line); and $\Delta x=0.01, \Delta t=0.00001$ are used. 
Figure 4.16 shows the results for the second set of initial condition i.e. $u(x, 0)=2 k^{2} \operatorname{sech}^{2}(k x)$. It can be seen that the curves for Figure 4.16(a), (b) and (c) still conserve mass, has no decrease in height and spread out consistently. More runtime is needed here in order to produce no oscillations to the curves.
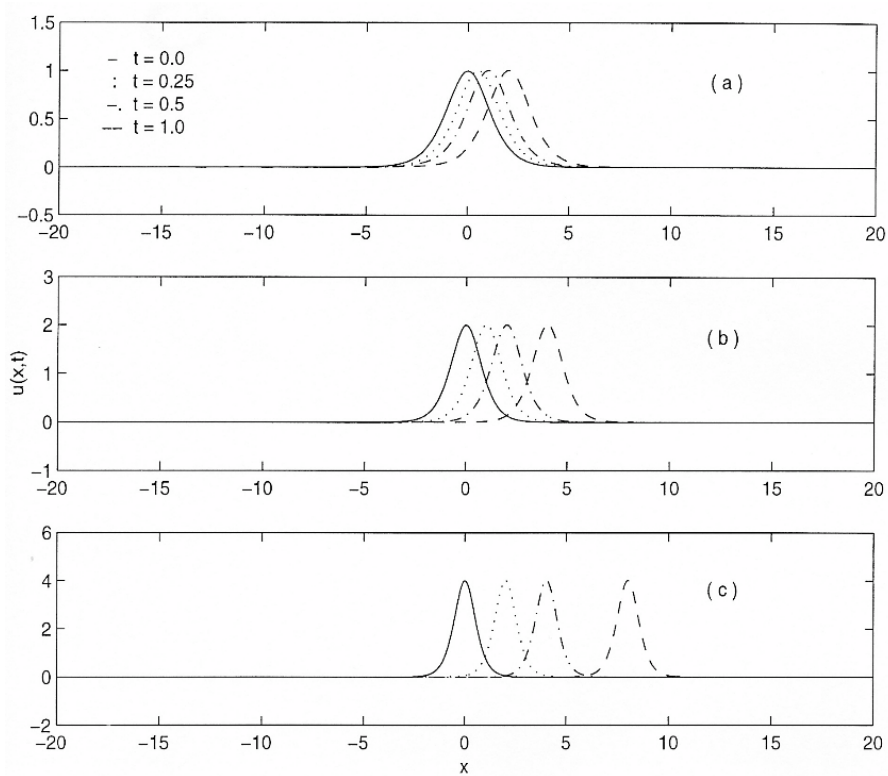

Figure 4.16. 1 - Soliton Solution for Walkley Scheme on the interval [-20, 20]

Note: Figure 4.13(a) amplitude $=1, \Delta x=0.1739, \Delta t=0.00001$; Figure 4.13(b) amplitude $=2, \Delta x=0.08, \Delta t=$ 0.000001 ; and Figure 4.13(c) amplitude $=4, \Delta x=0.05, \Delta t=0.0000001$.

Applying the third initial condition of equation (3.4) with two different sets of parameters, Figures 4.17(a) and (b) are produces. For the last figures of $4.17(\mathrm{a})$ and (b) the tow solitons move further apart and since periodic boundary condition on the interval $[-20,20]$ is used, they will collide in time and their original shapes will be preserved. Again, blips or humps are left in the middle but in reality it is non-existent.
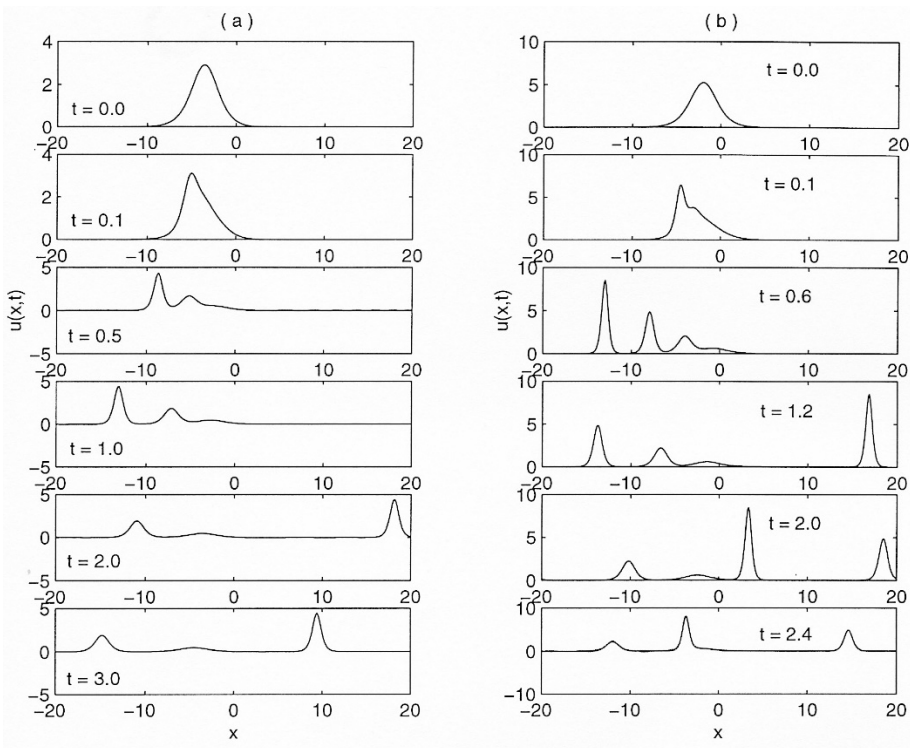

Figure 4.17. Separation into Two Soliton for Walkley Scheme when they are allowed to separate on the interval $[-20,20]$.

Note: Figure 4.17(a) Parameter 1: amplitudes $=\frac{1}{2}$ and 1, $\Delta x=0.12, \Delta t=0.0000001$; and Figure 4.17(b) Parameter 2: amplitudes $=\frac{1}{2}$ and $\frac{5}{2}, \Delta x=0.06, \Delta t=0.0000001$. 


\section{Conclusions and Remarks}

For this study, two types of Korteweg-de Vries equation were investigated (equations (2.2) and (3.1)) which represents the simplest possible nonlinear dispersive equations. The two types of equations were used as exact solutions of the initial condition. Not only the initial value problem for the cos and $\operatorname{sech}^{2}$ functions for the $1-$ Soliton solution were analysed, a Two-Soliton solution with different values of parameters was also studied. Periodic boundary condition were implemented for all the computations beginning at $t=0$ and ending at $t=T$.

According to the approach given by the initial conditions stated in Section 3, our main interest was to analyse which explicit scheme performs well when implemented to the $\mathrm{KdV}$ equation to produce the best soliton solution. We have concluded after comparing the four explicit schemes, the best scheme was the Zabusky and Kruskal scheme since it is a two-step scheme, which uses the explicit leapfrog finite difference scheme. Zabusky and Kruskal's scheme was good for low amplitudes and less running time was needed than the other three explicit schemes.

The use of all the explicit schemes leads to irregular solitons, which makes the wave pattern more and more unrealistic in the course of time. The Lax-Wendroff scheme and Walkley scheme tie for the second best scheme. However, more time was needed to run the codes so no unwanted oscillations were to be detected.

The scheme that performed the worst was no doubt the First Order Upwind Scheme. Since this scheme is of first order, it has a low order that dampens the amplitudes of each wave and with higher wave numbers it will be more severely affected. All the solitons in Figures 4.4, 4.5 and 4.6 numerically diffuse and eventually spread out through time. The diffusion was most noticeable in Figure 4.5(c) Although for second order schemes, i.e. Zabusky and Kruskal scheme and Lax-Wendroff scheme, there was not a drastic smearing of discontinuities as there was for the First Order Upwind Scheme, the solutions may be polluted by spurious oscillations.

All the schemes with the separation into two solitons as the initial condition i.e $u(x, t)=2 \frac{\partial^{2}}{\partial x^{2}}\left[\log _{e} f(x, t)\right]$, we observed that the blips or humps are prominent, as seen in Figures 4.6, 4.9, 4.14 and 4.17. These blips are non-zero effects due to numerical errors, which is also the consequence of dispersion.

In summary, we have reviewed and considered the four classical existing schemes for the Korteweg-de Vries equation for each of the four schemes presented in this paper. After comparing the four explicit schemes, the best scheme was the Zabusky and Kruskal scheme since it is a two-step scheme, which uses the explicit leapfrog finite difference scheme and was good for low amplitudes and less running time was needed than the other three explicit schemes. Furthermore, Katuhiko Goda (1975) proposed an implicit scheme that is unconditionally stable for the $\mathrm{KdV}$ equation. He also concluded that implicit schemes often gave better results than explicit schemes. Therefore, further study is necessary in finding the best scheme for the KdV equation.

\section{Acknowledgements}

The initial work of this study was carried out as part of Masitah Shahrill's Master study at the University of Reading. MS acknowledges the support and scholarship grant from The Government of His Majesty the Sultan and Yang Di-Pertuan Negara Brunei Darussalam. Substantial preparation of this paper was subsequently done by all co-authors (Masitah Shahrill, Maureen Maureen Siew Fang Chong \& Hajah Norhakimah Haji Mohd Nor).

\section{References}

Akdi, M., \& Sedra, M. B. (2013a). Numerical simulation of KdV equation. Advanced Studies in Theoretical Physics, 7(9), 407-418.

Akdi, M., \& Sedra, M. B. (2013b). Numerical KdV equation by the adomian decomposition method. American Journal of Modern Physics, 2(3), 111-115. http://dx.doi.org/10.11648/j.ajmp.20130203.13

Ascher, U. M., \& McLachlan, R. I. (2005). On symplectic and multisymplectic schemes for the KdV equation. Journal of Scientific Computing, 25(1/2), 83-104. http://dx.doi.org/10.1007/s10915-004-4634-6

Boussinesq, J. (1871). Théorie de l'intumescence liquide, applelée onde solitaire ou de translation, se propageant dans un canal rectangulaire. Comptes Rendus de l'Academie des Sciences, 72, 755-759.

Drazin, P. G., \& Johnson, R. S. (1989). Solitons: An Introduction. Cambridge University Press, Cambridge. http://dx.doi.org/10.1017/CBO9781139172059

Dutykh, D., Chhay, M., \& Fedele, F. (2013). Geometric numerical schemes for the KdV equation. Computational Mathematics and Mathematical Physics, 53(2), 221-236. http://dx.doi.org/10.1134/S0965542513020103 
Gardner, C. S., Greene, J. M., Kruskal, M. D., \& Miura, R. M. (1967). Method for solving the korteweg-de vries Equation, Physics Review Letter, 19, 1095-1097. http://dx.doi.org/10.1103/PhysRevLett.19.1095

Goda, K. (1975). On stability of some finite difference schemes for the korteweg-de vries equation. Journal of the Physics Society of Japan, 39(1), 229-236. http://dx.doi.org/10.1143/JPSJ.39.229

Hasegawa, A. (1990). Optical Solitons in Fibers. Springer-Verlag Berlin Heidelberg, New York. http://dx.doi.org/10.1007/978-3-662-09113-5

Kakutani, T., Kawahara, T., \& Taniuti, T. (1967). Nonlinear hydromagnetic solitary waves in a collision-free plasma with isothermal electron pressure. Journal of the Physical Society of Japan, 23(5), 1138-1149. http://dx.doi.org/10.1143/JPSJ.23.1138

Korteweg, D. J., \& DeVries, G. (1895). On the change of form and long waves advancing in a rectangular canal, and on a new type of long stationary waves. Philosophical Magazine, 39(5), 422-443. http://dx.doi.org/10.1080/14786449508620739

Lv, Z. Q., Xue, M., \& Wang, Y. S. (2011). A new multi-symplectic scheme for the KdV equation. Chinese Physics Letters, 28(6), 060205. http://dx.doi.org/10.1088/0256-307X/28/6/060205

Russell, J. S. (1845). Report on Waves. Report of the Fourteenth Meeting of the British Association for the Advancement of Science, York, September 1844, pp. 311-390, Plates XLVII-LVII.

Strutt, J. W. (1876). On waves. Philosophical Magazine Series 5, 1(4), 257-279. http://dx.doi.org/10.1080/14786447608639037

Sweby, P. K. (2000). C.T.M. Project 4, Hyperbolic Problems for M.Sc. Numerical Solutions for Differential Equations (1999-2000). University of Reading, Reading, United Kingdom.

Taha, T. R., \& Ablowitz, M. J. (1984). Analytical and numerical aspects of certain nonlinear evolution equations, III, numerical, korteweg-de vries equation. Journal of Computational Physics, 55(2), 231-253. http://dx.doi.org/10.1016/0021-9991(84)90003-2

Walkley, M. A. (1999). A Numerical Method for Extended Boussinesq Shallow-Water Wave Equations, Ph.d Thesis, School of Computer Studies, The University of Leeds, United Kingdom.

Wang, H. P., Wang, Y. S., \& Hu Y. Y. (2008). An explicit scheme for the KdV equation. Chinese Physics Letters, 25(7), 2335-2338. http://dx.doi.org/10.1088/0256-307X/25/7/002

Washimi, H., \& Taniuti, T. (1966). Propagation and ion-acoustic solitary waves of small amplitude. Physics Review Letter, 17(19), 996-998. http://dx.doi.org/10.1103/PhysRevLett.17.996

Zabusky, N. J. (1967). A Synergetic Approach to Problems of Nonlinear Wave Propagation and Interaction in Nonlinear Partial Differential Equations. In Proceedings of the Symposium on Nonlinear Partial Differential Equations (W. F. Ames, Ed.), pp. 223-258, New York. http://dx.doi.org/10.1016/B978-1-4831-9647-3.50019-4

Zabusky, N. J., \& Kruskal, M. D. (1965). Interaction of 'solitons' in a collisionless plasma and the recurrence of initial states. Physics Review Letter, 15(6), 240-243. http://dx.doi.org/10.1103/PhysRevLett.15.240

\section{Copyrights}

Copyright for this article is retained by the author(s), with first publication rights granted to the journal.

This is an open-access article distributed under the terms and conditions of the Creative Commons Attribution license (http://creativecommons.org/licenses/by/3.0/). 